\title{
On Some Boundedness and Convergence Properties of a Class of Switching Maps in Probabilistic Metric Spaces with Applications to Switched Dynamic Systems
}

\author{
M. De la Sen ${ }^{1}$ and A. Ibeas ${ }^{2}$ \\ ${ }^{1}$ Institute of Research and Development of Processes, University of the Basque Country, Campus of Leioa (Biscay), \\ P.O. Box 644, Bilbao, Barrio Sarriena, 48940 Leioa, Spain \\ ${ }^{2}$ Department of Telecommunications and Systems Engineering, Universitat Autònoma de Barcelona (UAB), Bellaterra, \\ Cerdanyola del Vallès, 08193 Barcelona, Spain
}

Correspondence should be addressed to A. Ibeas; asier.ibeas@uab.cat

Received 10 June 2015; Accepted 9 September 2015

Academic Editor: Wenguang Yu

Copyright (C) 2015 M. De la Sen and A. Ibeas. This is an open access article distributed under the Creative Commons Attribution License, which permits unrestricted use, distribution, and reproduction in any medium, provided the original work is properly cited.

\begin{abstract}
This paper investigates some boundedness and convergence properties of sequences which are generated iteratively through switched mappings defined on probabilistic metric spaces as well as conditions of existence and uniqueness of fixed points. Such switching mappings are built from a set of primary self-mappings selected through switching laws. The switching laws govern the switching process in between primary self-mappings when constructing the switching map. The primary self-mappings are not necessarily contractive but if at least one of them is contractive then there always exist switching maps which exhibit convergence properties and have a unique fixed point. If at least one of the self-mappings is nonexpansive or an appropriate combination given by the switching law is nonexpansive, then sequences are bounded although not convergent, in general. Some illustrative examples are also given.
\end{abstract}

\section{Introduction}

The background literature on fixed point theory and applications and associated convergence properties in metric spaces, Banach spaces, probabilistic metric spaces, Menger spaces, and some fuzzy-type versions is very abundant. See, for instance, [1-19] and the references therein. In particular, the theory focused on probabilistic metric spaces, including their specialization to Menger spaces, is also abundant. See, for instance, $[1-4,15,16,20]$ and the references therein. There are also studies in the graph framework for fixed point theory and problems of stability. See, for instance, [21, 22] and the references therein. On the other hand, fixed point theory has a wide range of applications, for instance, in the study of convergence of iterative schemes [17], in particular, of Mann and Jungck types or their many variants $[18,19]$, and in that of stability of dynamic systems and that of differential and difference equations. A particular class of real world applications refer to the stability of the so-called switched dynamic systems where a switching law assigns active parameterization for the dynamic system through time (or through an iterative discrete process) [23-27].

This paper investigates some boundedness and convergence properties of sequences which are generated through a class of switched mappings defined on probabilistic metric spaces, as well as conditions of existence and uniqueness of fixed points. The above switching mappings are defined via the selection as active of a set of primary self-mappings with the activation process governed by a "so-called" switching law. In this way, such switching laws govern the switching process in between primary self-mappings when constructing the switching map. The primary self-mappings are not necessarily contractive but if at least one of them is contractive then there always exist switching maps which exhibit convergence properties and have a unique fixed point. On the other hand, if at least one of the primary selfmappings is nonexpansive or an appropriate combination given by the switching law is nonexpansive, then sequences 
are bounded although not convergent, in general. Some illustrative examples are also discussed. Section 2 introduces $C_{\rho k}$ and $C_{k}$ classes of primary self-mappings in probabilistic metric spaces as well as associated upper- and lowerbounding constraints of the probability density of the built sequences. The above class allows the characterization of strict contractions as well as nonexpansive or expansive self-mappings in the probabilistic metric spaces. In parallel, some needed definitions are revisited while some preliminary results of convergence of sequences, Cauchy sequences, and boundedness of sequences in probabilistic metric spaces and in Menger spaces are obtained. Section 3 gives formalism in probabilistic metric spaces related to the switched maps defined via the activation of the primary self-mappings through switching laws. The obtained results for switched maps rely on boundedness and convergence of sequences in a probabilistic context.

\section{On $C_{\rho k}$ and $C_{k}$ Classes of Self-Mappings in Probabilistic Metric Spaces}

Let us define a probabilistic distance $\mathbf{F}: X \times X \rightarrow \Delta_{\mathbf{F}}$, where $X$ is a nonempty abstract set represented by $F_{x, y}$ for each $(x, y) \in X \times X$, where $\Delta_{\mathrm{F}}$ is a set of distribution functions. A distribution function $F \in \Delta_{\mathrm{F}}$ is a mapping $F: \mathbf{R} \rightarrow \mathbf{R}_{0+}$ which is nondecreasing and left-continuous with $\inf _{t \in \mathbf{R}} F(t)=$ 0 and $\sup _{t \in \mathbf{R}} F(t)=1$.

The ordered pair $(X, \mathbf{F})$ is a probabilistic metric (PM) space if for any $x, y, z \in X$ and all $t, s \in \mathbf{R}_{+}$the following conditions hold [1]:

(1) $F_{x, y}(t)=H(t) \Longleftrightarrow x=y$,

where $H \in \Delta_{\mathbf{F}}$ is defined by $H(t)= \begin{cases}0, & \text { if } t \leq 0 \\ 1, & \text { if } t>0\end{cases}$

(2) $F_{x, y}(t)=F_{y, x}(t)$;

(3) if $F_{x, y}(t)=1$,

$$
F_{y, z}(s)=1
$$

then $F_{x, z}(t+s)=1$.

The triplet $(X, \mathbf{F}, \Delta)$ is a Menger space where $(X, \mathbf{F})$ is a PMspace and $\Delta$ is a triangular norm which satisfies the inequality $F_{x, z}(t+s) \geq \Delta\left(F_{x, y}(t), F_{y, z}(s)\right), \forall x, y, z \in X, \forall t, s \in \mathbf{R}_{+}$.

Note that $F_{x, y}(0)=F_{x, y}(t)=0$ for $t \leq 0$ and $F_{x, y}(t)=$ $F_{x, y}\left(0^{+}\right)=1$ for $t>0$ if $x=y$ since $H \in \Delta_{\mathbf{F}}$ is nondecreasing and left-continuous. Note also that every metric space $(X, d)$ can be realized as a PM-space by taking $\mathbf{F}: X \times X \rightarrow \Delta_{\mathbf{F}}$ being defined by $F_{x, y}(t)=H(t-d(x, y))$ for all $x, y \in X$ [1-4]. In the following, $D_{+}$is the space of all mappings $F$ : $\mathbf{R} \rightarrow[0,1]$ which are left-continuous and nondecreasing with $F(0)=0$ and $\ell^{-} F(+\infty)=1$. The space $D_{+}$is partially ordered by the usual pointwise ordering of functions; namely, $F \leq G$ if and only if $F(t) \leq G(t), \forall t \in \mathbf{R}$, and its maximal element is the distribution $H(t)$ [4].
Definition 1. Let $(X, \mathbf{F})$ be a PM-space. A mapping $T: X \rightarrow$ $X$ is said to be of $C_{k}$-class for some function $k: X \times X \rightarrow \mathbf{R}_{+}$ if

$$
F_{T x, T y}(t) \geq F_{x, y}\left(k^{-1}(x, y) t\right) ; \quad \forall x, y \in X, \forall t \in \mathbf{R}_{+} .
$$

Definition 2. Let $(X, \mathbf{F})$ be PM-space. A mapping $T: X \rightarrow X$ is said to be of $C_{\rho k}$-class for some functions $\rho, k: X \times X \rightarrow$ $\mathbf{R}_{+}$if

$$
\begin{array}{r}
F_{x, y}\left(\rho^{-1}(x, y) t\right) \geq F_{T x, T y}(t) \geq F_{x, y}\left(k^{-1}(x, y) t\right) \\
\forall x, y \in X, \forall t \in \mathbf{R}_{+},
\end{array}
$$

where the functions $\rho, k: X \times X \rightarrow \mathbf{R}_{+}$satisfy $\rho(x, y) \leq$ $k(x, y), \forall x, y \in X$.

Note that if $T: X \rightarrow X$ is of $C_{\rho k}$-class, then it is also of $C_{k}$-class. Note also that $T: X \rightarrow X$ is nonexpansive if it is of $C_{k}$-class with $\sup _{x, y \in X} k(x, y) \leq 1$ and, in particular, a probabilistic strict contraction if it is of $C_{k}$-class with $\sup _{x, y \in X} k(x, y)<1$. Also, if $T: X \rightarrow X$ is of $C_{\rho k}$-class with $\sup _{x, y \in X} k(x, y) \leq 1\left(\sup _{x, y \in X} k(x, y)<1\right)$, then it is nonexpansive (probabilistic strictly contractive) [1-4]. If $T$ : $X \rightarrow X$ is of $C_{\rho k}$-class with $1<\inf _{x, y \in X} \rho(x, y) \leq$ $\inf _{x, y \in X} k(x, y)$, then it is expansive [1-4]. If there is some $\rho: X \times X \rightarrow \mathbf{R}_{+}$with $\inf _{x, y \in X} \rho(x, y)>1$ such that

$$
F_{x, y}\left(\rho^{-1}(x, y) t\right) \geq F_{T x, T y}(t) ; \quad \forall x, y \in X, \forall t \in \mathbf{R}_{+},
$$

then $T: X \rightarrow X$ is expansive (even if $T: X \rightarrow X$ is not of $C_{\rho k}$-class for some $k: X \times X \rightarrow \mathbf{R}_{+}$subject to $1<$ $\left.\inf _{x, y \in X} \rho(x, y) \leq \inf _{x, y \in X} k(x, y)\right)$.

The following technical result follows.

Lemma 3. The following properties hold:

(i) Let $(X, \mathbf{F})$ be a PM-space and let $T: X \rightarrow X$ be a mapping of $C_{\rho k}$-class. Consider the sequences $\left\{x_{n}\right\} \subseteq$ $X$ and $\left\{y_{n}\right\} \subseteq X$ built by $x_{n+1}=T x_{n}, y_{n+1}=T y_{n}$, $\forall n \in \mathbf{Z}_{0+}$ with $x_{0}=x, y_{0}=y$ for some given $x, y \in X$. Then,

$$
\begin{gathered}
F_{x, y}\left(\prod_{i=0}^{n-1}\left[\rho_{i}^{-1}(x, y)\right] t\right) \geq F_{T^{n} x, T^{n} y}(t) \\
\geq F_{x, y}\left(\prod_{i=0}^{n-1}\left[k_{i}^{-1}(x, y)\right] t\right)
\end{gathered}
$$

where $k_{n}(x, y)=k\left(T^{n} x, T^{n} y\right), \rho_{n}(x, y)=$ $\rho\left(T^{n} x, T^{n} y\right), \forall n \in \mathbf{Z}_{0+}$.

(ii) If $T: X \rightarrow X$ is of $C_{k}$-class, then $F_{T^{n} x, T^{n} y}(t) \geq$ $F_{x, y}\left(\prod_{i=0}^{n-1}\left[k_{i}^{-1}(x, y)\right] t\right), \forall n \in \mathbf{Z}_{0+}$.

(iii) If $T: X \rightarrow X$ is a mapping of either $C_{k}$-class or $C_{\rho k^{-}}$ class with $\lim _{n \rightarrow \infty} \prod_{i=0}^{n}\left[k_{i}(x, y)\right]=0$ for the given $x, y \in X$, then $\lim _{n \rightarrow \infty} F_{T^{n} x, T^{n} y}(t)=1, \forall t \in \mathbf{R}_{+}$. 
(iv) If $T: X \rightarrow X$ is a mapping of $C_{\rho k^{-c l a s s} \text { with }} \beta_{n}=$ $\prod_{i=0}^{n-1}\left[\rho_{i}^{-1}(x, y)\right], \alpha_{n}=\prod_{i=0}^{n-1}\left[k_{i}^{-1}(x, y)\right], \forall n \in \mathbf{Z}_{0+}$, and

$$
\begin{aligned}
& \alpha=\alpha(x, y)=\liminf _{n \rightarrow \infty} \alpha_{n}, \\
& \beta=\beta(x, y)=\limsup _{n \rightarrow \infty} \beta_{n}
\end{aligned}
$$

are in $c l \mathbf{R}_{0+}=\mathbf{R}_{0+} \cup\{+\infty\}$ (i.e., $c \mid \mathbf{R}_{0+}$ is the closure of $\mathbf{R}_{0+}$, i.e., the extended nonnegative real semiline) for the given $x, y \in X$, then

$$
\begin{gathered}
F_{x, y}\left(\beta_{n} t\right) \geq F_{T^{n} x, T^{n} y}(t) \geq F_{x, y}\left(\alpha_{n} t\right) ; \\
\forall n \in \mathbf{Z}_{0+} \forall t \in \mathbf{R}_{+}, \\
F_{x, y}(\beta t) \geq \limsup _{n \rightarrow \infty} F_{T^{n} x, T^{n} y}(t) \geq \liminf _{n \rightarrow \infty} F_{T^{n} x, T^{n} y}(t) \\
\geq F_{x, y}(\alpha t) ; \quad \forall t \in \mathbf{R}_{+} .
\end{gathered}
$$

If $T: X \rightarrow X$ is a mapping of $C_{k}$-class, then $\liminf _{n \rightarrow \infty} F_{T^{n} x, T^{n} y}(t) \geq F_{x, y}(\alpha t), \forall t \in \mathbf{R}_{+}$.

Proof. It follows recursively from (3) with $x_{n+1}=T x_{n}, y_{n+1}=$ $T y_{n}, \forall n \in \mathbf{Z}_{0+}$, with $x_{0}=x, y_{0}=y$ for the given $x, y \in X$ that

$$
\begin{gathered}
F_{x, y}\left(\rho_{0}^{-1}(x, y) t\right) \geq F_{T x, T y}(t) \geq F_{x, y}\left(k_{0}^{-1}(x, y) t\right), \\
F_{x, y}\left(\rho_{0}^{-1}(x, y) \rho_{1}^{-1}(x, y) t\right) \geq F_{T x, T y}\left(\rho_{1}^{-1}(x, y) t\right) \\
\geq F_{T^{2} x, T^{2} y}(t) \geq F_{T x, T y}\left(k_{1}^{-1}(x, y) t\right) \\
\geq F_{x, y}\left(k_{0}^{-1}(x, y) k_{1}^{-1}(x, y) t\right), \\
\vdots \\
F_{x, y}\left(\prod_{i=0}^{n-1}\left[\rho_{i}^{-1}(x, y)\right] t\right) \geq F_{T^{n} x, T^{n} y}(t) \\
\geq F_{x, y}\left(\prod_{i=0}^{n-1}\left[k_{i}^{-1}(x, y)\right] t\right) ;
\end{gathered}
$$$$
\forall x, y \in X, \forall t \in \mathbf{R}_{+}, \forall n \in \mathbf{Z}_{0+} .
$$

Property (i) has been proved and the proof of Property (ii) follows directly by just using the lower-bounding part of the recursion. Property (iii) follows since $\lim _{n \rightarrow \infty} \prod_{i=0}^{n-1}\left[k_{i}^{-1}(x\right.$, $y)]=+\infty$; then, $\lim _{n \rightarrow \infty}\left(\prod_{i=0}^{n-1}\left[k_{i}^{-1}(x, y)\right]\right) t=+\infty, \forall t \in$ $\mathbf{R}_{+}$, and the conditions that $F_{x, y}(t)$ is nondecreasing in the argument $t$ and $\sup _{t \in \mathbf{R}_{+}} F_{x, y}(t)=\lim \sup _{t \rightarrow \infty} F_{x, y}(t)=1$ lead

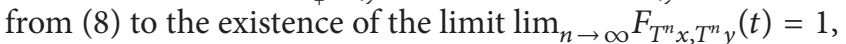
$\forall t \in \mathbf{R}_{+}$. Property (iv) is proved closely to Property (iii) by first getting (7a) and (7b) directly from the definitions of $\beta_{n}$, $\alpha_{n}, \beta$, and $\alpha, \forall n \in \mathbf{Z}_{0+}$.

The subsequent example illustrates that Lemma 3 is useful for the characterization of probabilities which can be less than one (i.e., the probabilistic certainty) through lower-bounds and upper-bounds in probabilistic metric spaces.
Example 4. Let us consider the metric space $(X, d)$ with $F$ : $X \times X \rightarrow \Delta_{F}$ being defined by $F_{x, y}(t)=H(t-d(x, y))$ for all $x, y \in X$ for the distribution function $H_{a b} \in \Delta_{F}$ defined by:

$$
H_{a b}(t)= \begin{cases}a(t), & \text { if } t \leq 0, \\ b(t), & \text { if } t>0\end{cases}
$$

for some left-continuous nondecreasing functions $a, b$ : $\mathbf{R}_{0+} \rightarrow[0,1]$ with

$$
b(x, y, t) \geq a(x, y, t)=a(x, y,-t) ; \quad \forall t \in \mathbf{R},
$$

$$
\begin{aligned}
& \lim _{t \rightarrow-\infty} a(x, y, t)=0, \\
& \lim _{t \rightarrow-\infty} b(x, y, t)=1 .
\end{aligned}
$$

Assume also that $a: \mathbf{R}_{0+} \rightarrow[0,1]$ is everywhere lowersemicontinuous and $b: \mathbf{R}_{0+} \rightarrow[0,1]$ is everywhere uppersemicontinuous. Then,

$$
\begin{aligned}
& H_{a b}(\alpha t-d(x, y)) \\
& = \begin{cases}a(\alpha t-d(x, y)), & \text { if } t \leq \frac{d(x, y)}{\alpha}, \\
b(\alpha t-d(x, y)), & \text { if } t>\frac{d(x, y)}{\alpha},\end{cases} \\
& \forall x, y \in X
\end{aligned}
$$

with $a\left(x, y, 0^{-}\right)=a\left(x, y, 0^{+}\right)=b\left(x, y, 0^{-}\right)=0$, $\lim _{t \rightarrow-\infty} a(x, y, t)=0$, and $\lim _{t \rightarrow+\infty} b(x, y, t)=1, \forall x, y \in$ $X$. Assume following Lemma 3(iv) that $\beta=\beta(x, y)=$ $\lim \sup _{n \rightarrow \infty} \beta_{n}$ and $\alpha=\alpha(x, y)=\liminf _{n \rightarrow \infty} \alpha_{n}$ with $\beta_{n}=$ $\beta_{n}(x, y)=\prod_{i=0}^{n-1}\left[\rho_{i}^{-1}(x, y)\right], \alpha_{n}=\alpha_{n}(x, y)=\prod_{i=0}^{n-1}\left[k_{i}^{-1}(x\right.$, $y)], \forall n \in \mathbf{Z}_{0+}$. Note that $\alpha, \beta, \alpha_{n}$, and $\beta_{n}$ are allowed to be dependent on $x, y$. Then, if $T: X \rightarrow X$ is a mapping of $C_{\rho k}{ }^{-}$ class so that (7a) and (7b) of Lemma 3 hold, one gets for any given $x, y \in X$

$$
\begin{aligned}
b\left(\beta_{n} t-d(x, y)\right) & =F_{x, y}\left(\beta_{n} t\right) \\
& =H_{a b}\left(\beta_{n} t-d(x, y)\right) \\
& \geq \limsup _{n \rightarrow \infty} F_{T^{n} x, T^{n} y}(t) \\
& =\limsup _{n \rightarrow \infty} H_{a b}\left(t-d\left(T^{n} x, T^{n} y\right)\right) \\
& \geq \liminf _{n \rightarrow \infty} F_{T^{n} x, T^{n} y}(t) \\
& =\liminf _{n \rightarrow \infty} H_{a b}\left(t-d\left(T^{n} x, T^{n} y\right)\right) \\
& \geq F_{x, y}\left(\alpha_{n} t\right)=b\left(\alpha_{n} t-d(x, y)\right) \\
& =H_{a b}\left(\alpha_{n} t-d(x, y)\right) ;
\end{aligned}
$$




$$
\begin{aligned}
b(\beta t-d(x, y)) & =F_{x, y}(\beta t)=H_{a b}(\beta t-d(x, y)) \\
& \geq \limsup _{n \rightarrow \infty} F_{T^{n} x, T^{n} y}(t) \\
& =\limsup _{n \rightarrow \infty} H_{a b}\left(t-d\left(T^{n} x, T^{n} y\right)\right) \\
& \geq \liminf _{n \rightarrow \infty} F_{T^{n} x, T^{n} y}(t) \\
& =\liminf _{n \rightarrow \infty} H_{a b}\left(t-d\left(T^{n} x, T^{n} y\right)\right) \\
& \geq F_{x, y}(\alpha t)=b(\alpha t-d(x, y)) \\
& =H_{a b}(\alpha t-d(x, y))
\end{aligned}
$$

from (7a) and (7b). Thus, one gets for any given $x, y \in X$ the following:

(a) If $t_{n}>d(x, y) / \alpha_{n}$ for some given $n \in \mathbf{Z}_{0+}$, then, since $t_{n}>d(x, y) / \beta_{n}$ as well, one gets

$$
\begin{aligned}
b\left(\beta_{n} t_{n}-d(x, y)\right) & \geq F_{T^{n} x, T^{n} y}\left(t_{n}\right) \\
& \geq b\left(\alpha_{n} t_{n}-d(x, y)\right)
\end{aligned}
$$

and if $t>d(x, y) / \alpha$ since $t>d(x, y) / \beta$, then

$$
\begin{aligned}
b(\beta t-d(x, y)) & \geq \limsup _{n \rightarrow \infty} F_{T^{n} x, T^{n} y}(t) \\
& \geq \liminf _{n \rightarrow \infty} F_{T^{n} x, T^{n} y}(t) \\
& \geq b(\alpha t-d(x, y))
\end{aligned}
$$

and $\exists \lim _{t \rightarrow+\infty} \lim _{n \rightarrow \infty} F_{T^{n} x, T^{n} y}(t)=1$ if $\alpha>0$ since the above superior and inferior limits equalize unity.

(b) If $d(x, y) / \beta_{n}<t \leq d(x, y) / \alpha_{n}$, then

$$
\begin{aligned}
H_{a b}\left(\alpha_{n} t-d(x, y)\right) & =a\left(\alpha_{n} t-d(x, y)\right) \\
& \leq \liminf _{n \rightarrow \infty} F_{T^{n} x, T^{n} y}(t) \\
& \leq \limsup _{n \rightarrow \infty} F_{T^{n} x, T^{n} y}(t) \\
& \leq H_{a b}\left(\beta_{n} t-d(x, y)\right) \\
& =b\left(\beta_{n} t-d(x, y)\right) .
\end{aligned}
$$

If, furthermore, $\beta=\alpha>0$, then $\exists \lim _{t \rightarrow+\infty} \lim _{n \rightarrow \infty} F_{T^{n} x, T^{n} y}(t)=1$. If, in addition, $\alpha=+\infty$, then $\beta=+\infty, a(t)=0$, and $b(t)=1$, $\forall t \in \mathbf{R}_{+}$; then $\exists \lim _{n \rightarrow \infty} F_{T^{n} x, T^{n} y}(t)=1, \forall t \in \mathbf{R}_{+}$, which is the basic convergence suitable property in probabilistic metric spaces for probabilistic strictly contractive mappings in the existing literature. Note that this case includes the case under Lemma 3(iii) when $\lim _{n \rightarrow \infty} \prod_{i=0}^{n}\left[k_{i}(x, y)\right]=0$ leading to

$$
\begin{aligned}
\lim _{n \rightarrow \infty} F_{T^{n} x, T^{n} y}(t) & =\lim _{n \rightarrow \infty} H_{a b}\left(t-d\left(T^{n} x, T^{n} y\right)\right) \\
& \geq F_{x, y}(+\infty)=H(+\infty)=1 ;
\end{aligned}
$$

that is, $\lim _{n \rightarrow \infty} F_{T^{n} x, T^{n} y}(t)=1, \forall x, y \in X, \forall t \in \mathbf{R}_{+}$, or, in other words, for any distance $d(x, y)$ from a given $x \in X$ to a given $y \in X, \lim _{n \rightarrow \infty} d\left(T^{n} x, T^{n} y\right)=$ 0 .

(c) If $t \leq d(x, y) / \beta_{n}$, then $\liminf _{n \rightarrow \infty} F_{T^{n} x, T^{n} y}(t) \geq$ $a\left(\beta_{n} t-d(x, y)\right)$.

(d) Now, assume that $T: X \rightarrow X$ is a mapping of $C_{\rho k^{-}}$ class with $1<\inf _{x, y \in X} \rho(x, y) \leq \inf _{x, y \in X} k(x, y)$ and then $\beta=\alpha=0$; that is, the mapping is expansive. Then, if $t \rightarrow+\infty$ implying that $t>d(x, y) / \beta$ (and also $t>d(x, y) / \beta$ since $\alpha=\beta=0)$, one concludes from (13b) that

$$
\begin{aligned}
& \inf _{t>d(x, y) / \beta_{n}} F_{T^{n} x, T^{n} y}(t)=1, \\
& \sup _{t \leq d(x, y) / \beta_{n}} F_{T^{n} x, T^{n} y}(t)<1 ;
\end{aligned}
$$

$$
\forall x, y(\neq x) \in X
$$

$$
\begin{aligned}
& \lim _{n \rightarrow+\infty} \inf _{t>d(x, y) / \beta_{n}} F_{T^{n} x, T^{n} y}(t)=1, \\
& \lim _{n \rightarrow+\infty} \sup _{t \leq d(x, y) / \beta_{n}} F_{T^{n} x, T^{n} y}(t)<1 ;
\end{aligned}
$$

$$
\forall x, y(\neq x) \in X
$$

since $\left\{\alpha_{n}\right\} \rightarrow 0$ and $\left\{\beta_{n}\right\} \rightarrow 0$. The constraints (13a) still hold for each $n \in \mathbf{Z}_{0+}$ such that $t_{n}>d(x, y) / \alpha_{n}$ but the sequence $\left\{t_{n}\right\}$ diverges to $+\infty$ while $\left\{\alpha_{n}\right\} \rightarrow 0$ and $\left\{\beta_{n}\right\} \rightarrow 0$.

Example 5. Assume that $\alpha=\beta=\alpha_{n}=\beta_{n}=1, \forall n \in \mathbf{Z}_{0+}$, independent of $x, y \in X$. Then, $T: X \rightarrow X$ is of $C_{11}$-class and nonexpansive but also probabilistic noncontractive. If $t>$ $d(x, y)$, then one gets from (13a) and (13b)

$$
\begin{aligned}
& F_{T^{n} x, T^{n} y}(t)=F_{x, y}(t)=b(t-d(x, y)), \\
& \lim _{t \rightarrow+\infty} \lim _{n \rightarrow \infty} F_{T^{n} x, T^{n} y}(t)=\lim _{t \rightarrow+\infty} b(t-d(x, y)) \\
& \quad=\lim _{t \rightarrow+\infty} H_{a b}(t)=1 ;
\end{aligned}
$$

$\forall x, y \in X$

Assume instead that $\alpha_{n_{k}}=\beta_{n_{k}}=1$ for some sequence $\left\{n_{k}\right\} \subseteq$ $\mathbf{Z}_{0+}, \forall k \in \mathbf{Z}_{0+}$. Then, if $t_{n_{k}}>d(x, y)$, one has for any $x, y \in X$ that

$$
\begin{gathered}
F_{T^{n} x, T^{n} y}\left(t_{n_{k}}\right)=b\left(t_{n_{k}}-d(x, y)\right) \\
\text { if } t_{n_{k}}>d(x, y) ; \\
b\left(\alpha_{n} t-d(x, y)\right) \leq F_{T^{n} x, T^{n} y}(t) \leq b\left(\beta_{n} t-d(x, y)\right), \\
\forall t \in\left(t_{n_{k}}, t_{n_{k+1}}\right], \forall n \in\left(n_{k}, n_{k+1}\right] \text { if } t>\frac{d(x, y)}{\beta_{n}},
\end{gathered}
$$


which simplifies as $b\left(\alpha_{n} t-d(x, y)\right) \leq F_{T^{n} x, T^{n} y}(t) \leq b\left(\beta_{n} t-\right.$ $d(x, y)), \forall n \in \mathbf{Z}_{0+}$, if $t>d(x, y) / \beta_{n}$ :

$$
\begin{gathered}
\lim _{t \rightarrow+\infty} \lim _{k \rightarrow \infty} F_{T^{n_{k} x, T^{n_{k}} y}}(t)=\lim _{k \rightarrow \infty} b\left(t_{n_{k}}-d(x, y)\right) \\
=\lim _{t \rightarrow+\infty} b(t-d(x, y))=\lim _{k \rightarrow \infty} H_{a b}\left(t_{n_{k}}\right)=1 .
\end{gathered}
$$
result.

From Lemma 3(iii), one gets directly the subsequent

Proposition 6. Let $(X, \mathbf{F})$ be a PM-space and let $T: X \rightarrow$ $X$ be a mapping of either $C_{k}$-class or $C_{\rho k}$-class and there is a strictly increasing sequence of nonnegative integers $\left\{n_{k}\right\}$ fulfilling $\lim _{k \rightarrow \infty} \sup \left(n_{k+1}-n_{k}\right)<+\infty$ such that, for some given $x, y \in X, \prod_{i=n_{k}}^{n_{k}+n_{k+1}}\left[k_{i}(x, y)\right]<1, \forall k \in \mathbf{Z}_{0+}$; then $\lim _{n \rightarrow \infty} F_{T^{n} x, T^{n} y}(t)=1, \forall t \in \mathbf{R}_{+}$.

Proof. It follows from Lemma 3 that if $\prod_{i=n_{k}}^{n_{k}+n_{k+1}}\left[k_{i}(x\right.$, $y)]<1, \forall k \in \mathbf{Z}_{0+}$, then $\lim _{k \rightarrow \infty} \prod_{i=0}^{\sum_{i=0}^{k} n_{i}}\left[k_{i}(x, y)\right]=0$, $\lim _{k \rightarrow \infty} \prod_{i=0}^{\sum_{i=0}^{k} n_{i}+n}\left[k_{i}(x, y)\right]=0, \lim _{k \rightarrow \infty} \prod_{i=0}^{\sum_{i=0}^{k} n_{i}+n}\left[k_{i}^{-1}(x\right.$, $y)]=\infty, \forall n \in\left(n_{k}, n_{k+1}\right], \forall k \in \mathbf{Z}_{0+}$, and then $\lim _{n \rightarrow \infty} \prod_{i=0}^{n}\left[k_{i}^{-1}(x, y)\right]=\infty$.

Note that Proposition 6 includes as a particular case that of probabilistic strict contractions $T: X \rightarrow X$ which are then mappings of $C_{k}$-class with $0<\alpha^{-1}=\sup _{x, y \in X} k(x, y)<1$.

Definition 7 (see [2]). Let $(X, \mathbf{F})$ be a PM-space and $A$ a nonempty subset of $X$. The probabilistic diameter of $A$ is a mapping $D_{A}: \quad \mathbf{R}_{0+} \rightarrow[0,1]$ defined by $D_{A}(z)=$ $\sup _{t<z} \inf _{x, y \in A} F_{x, y}(t)$.

Definition 8 (see $[2,4])$. Let $(X, \mathbf{F})$ be a PM-space and $A$ a nonempty subset of $X$. The nonempty set $A$ is said to be probabilistically bounded if $\sup _{z \in \mathbf{R}_{0+}} D_{A}(z)=1$, that is, if the supremum of its probabilistic diameter $D_{A} \in D_{+}$.

We can define the set unboundedness as the concept opposite to Definition 8 as follows.

Definition 9 (see $[2,4])$. Let $(X, \mathbf{F})$ be a PM-space and $A$ a nonempty subset of $X$. The nonempty set $A$ is said to be probabilistically unbounded if $\sup _{z \in \mathbf{R}_{0+}} D_{A}(z)<1$, that is, if $D_{A} \notin D_{+}$.

The boundedness and unboundedness of sequences $\left\{x_{n}\right\} \subseteq X$ can be easily defined as supported by Definitions 8 and 9 as follows.

Definition 10 (see $[2,4])$. Let $(X, \mathbf{F})$ be a PM-space. The sequence $\left\{x_{n}\right\} \subseteq X$ is probabilistically bounded if $\sup _{z \in \mathbf{R}_{0+}}$ $\sup _{t<z} \inf _{n, m \in \mathbf{Z}_{0+}} \inf _{x_{n}, x_{m} \in X} F_{x_{n}, x_{m}}(t)=1$.
Definition 11 (see [1]). Let $(X, \mathbf{F})$ be a PM-space. Then, the sequence $\left\{x_{n}\right\} \subseteq X$ is

(1) probabilistically convergent to a point $x \in X$, denoted by $\left\{x_{n}\right\} \rightarrow x$, if for every $\varepsilon \in \mathbf{R}_{+}$and $\lambda \in(0,1)$ there exists some $N=N(\varepsilon, \lambda) \in \mathbf{Z}_{0+}$ such that

$$
F_{x_{n}, x}(\varepsilon)>1-\lambda ; \quad \forall n\left(\in \mathbf{Z}_{0+}\right) \geq N
$$

(2) Cauchy if for every $\varepsilon \in \mathbf{R}_{+}$and $\lambda \in(0,1)$ there exists some $N=N(\varepsilon, \lambda) \in \mathbf{Z}_{0+}$ such that

$$
F_{x_{n}, x_{m}}(\varepsilon)>1-\lambda ; \quad \forall n, m\left(\in \mathbf{Z}_{0+}\right) \geq N .
$$

A PM-space $(X, \mathbf{F})$ is complete if every Cauchy sequence is probabilistically convergent.

Proposition 12. Let $(X, \mathbf{F})$ be a PM-space. Then,

(1) $\left\{x_{n}\right\}(\rightarrow x) \subseteq X$ for some $x \in X$ if and only if the following limit exists: $\lim _{\varepsilon \rightarrow 0^{+}} \lim _{n \rightarrow \infty} F_{x_{n}, x}(\varepsilon)=1$;

(2) $\left\{x_{n}\right\} \subseteq X$ is a Cauchy sequence if and only if $\lim _{\varepsilon \rightarrow 0^{+}} \lim _{n \rightarrow \infty} F_{x_{n}, x_{n+m}}(\varepsilon)=1, \forall m \in \mathbf{Z}_{0+}$.

Proof. If $\left\{x_{n}\right\} \rightarrow x$, then there exists some $N=N(\varepsilon, \lambda) \in$ $\mathbf{Z}_{0+}$ such that $F_{x_{n}, x}(\varepsilon)>1-\lambda, \forall n\left(\in \mathbf{Z}_{0+}\right) \geq N$, for every $\varepsilon \in \mathbf{R}_{+}$and $\lambda \in(0,1)$. Thus, since $0 \leq F_{x_{n}, x}(\varepsilon) \leq 1, \forall n \in$ $\mathbf{Z}_{0+}$, then, by taking $\lambda \rightarrow 0^{+}$, one gets $\lim _{n \rightarrow \infty} F_{x_{n}, x}\left(0^{+}\right)=$ $\lim _{\varepsilon \rightarrow 0^{+}} \lim _{n \rightarrow \infty} F_{x_{n}, x}(\varepsilon)=1$.

Conversely, if $\lim _{n \rightarrow \infty} F_{x_{n}, x}\left(0^{+}\right)=1$, then for every $\varepsilon \in \mathbf{R}_{+}$ and $\lambda \in(0,1)$ there exists some $N=N(\varepsilon, \lambda) \in \mathbf{Z}_{0+}$ such that $F_{x_{n}, x}(\varepsilon)>1-\lambda, \forall n\left(\in \mathbf{Z}_{0+}\right) \geq N$; thus $\left\{x_{n}\right\} \rightarrow x$. Assume that this is not true. Thus, there is some subsequence $\left\{x_{n_{k}}\right\} \subseteq\left\{x_{n}\right\}$ such that $F_{x_{n_{k}}, x}(\varepsilon) \leq 1-\lambda$ for some $\varepsilon \in \mathbf{R}_{+}$and $\lambda \in(0,1)$ while $\lim _{n \rightarrow \infty} F_{x_{n}, x}(\varepsilon)=\lim _{n \rightarrow \infty} F_{x_{n}, x}\left(0^{+}\right)=1$ for any $\varepsilon \in \mathbf{R}_{+}$since $F_{x_{n}, x}(\varepsilon)$ is nondecreasing in the argument $\varepsilon$ and one gets the following contradiction for some $\lambda \in(0,1)$ :

$$
\begin{aligned}
1-\lambda & \geq \lim _{k \rightarrow \infty} F_{x_{n_{k}}, x}(\varepsilon)=\lim _{n \rightarrow \infty} F_{x_{n}, x}(\varepsilon) \\
& =\lim _{n \rightarrow \infty} F_{x_{n}, x}\left(0^{+}\right)=1 .
\end{aligned}
$$

Proposition 12(1) has been proved. The proof of Proposition 12(2) is very close and it is omitted.

Proposition 13. Let $(X, F)$ be a PM-space. Then, the sequence $\left\{x_{n}\right\} \subseteq X$ is probabilistically bounded if and only if $D_{a M\left\{x_{n}\right\}}=$ 1 , where $D_{a M\left\{x_{n}\right\}}=\sup _{z \in \mathbf{R}_{0+}} D_{a\left\{x_{n}\right\}}(z)$ with $D_{a\left\{x_{n}\right\}}(z)=$ $\sup _{t<z} \inf _{x_{n} \in X} F_{a, x_{n}}(t)$ for some $a \in X$, that is, if and only if $D_{a M\left\{x_{n}\right\}} \in D_{+}$for some $a \in X$.

Proof. If $X$ is bounded, then the result is direct for any sequence $S=\left\{x_{n}\right\} \subseteq X$. Assume that $X$ is not bounded and proceed by contradiction by assuming that $S \subseteq X$ is probabilistically bounded and $D_{a M S} \notin D_{+}$for some $a \in X$. On the other hand, since $S \subseteq X$ is probabilistically bounded, then, for all $x_{k}, x_{m} \in S$, there is $t_{0}=t_{0}\left(x_{k}, x_{m}\right) \in \mathbf{R}_{0+}$ such that $F_{x_{k}, x_{m}}(t)=1, \forall t\left(\in \mathbf{R}_{0+}\right)>t_{0}$. Since $D_{a M S} \notin D_{+}$, there is $x_{k} \in S$ such that $F_{a, x_{k}}(t)<1, \forall t\left(\in \mathbf{R}_{0+}\right)>t_{01}$, and some 
$t_{01}=t_{01}\left(x_{k}, a\right) \in \mathbf{R}_{0+}$. This implies from the contrapositive equivalent logic proposition to the third property of (1) of $(X, F)$ being a PM-space that either $F_{x_{k}, x_{m}}(t / 2)<1$, and then the sequence $S \subseteq X$ is not probabilistically bounded, $\forall t(\epsilon$ $\left.\mathbf{R}_{0+}\right)>t_{01}$ (a contradiction), or $F_{a, x_{m}}(t / 2)<1, \forall t\left(\in \mathbf{R}_{0+}\right)>$ $t_{01}$, for any given $x_{m} \in S$, and then $\limsup _{t \rightarrow+\infty} F_{a, x_{m}}(t / 2)<$ 1 for some fixed $a, x_{m} \in X$. Now, assume that, for all $a \in X$ such that $a \notin S, \lim \sup _{t \rightarrow+\infty} F_{a, x_{m}}(t / 2)<1$. Thus, the subset $A_{a}=\left\{a, x_{m}\right\}$ of $X, \forall a \in X$, is unbounded since its probabilistic diameter is less than one; that is, $D_{A_{a}} \notin D_{+}, \forall a \in$ $X$, and then the sequence $S$ is probabilistically unbounded, again a contradiction. It has been proved that if $S \subset X$ is bounded, then $D_{a M S} \in D_{+}$for some $a \in X \cap \bar{S}$, where $\bar{S}$ is the complementary to $S$ in $X$. It remains to prove that if $D_{a M S} \in D_{+}$for some $a \in X \cap \bar{S}$, then $S \subset X$ is probabilistically bounded. Since $D_{a M S} \in D_{+}$, then $F_{a, x_{k}}(t)=1, \forall t\left(\in \mathbf{R}_{0+}\right)>t_{0}$, for some $t_{0}=t_{0}\left(a, x_{k}\right) \in \mathbf{R}_{0+}$ and all $x_{k} \in S$. It follows from the third property of (1) that $F_{x_{n}, x_{m}}(t)=1, \forall t\left(\in \mathbf{R}_{0+}\right)>2 t_{0}$, $\forall x_{n}, x_{m} \in S$. Thus, $S \subset X$ is probabilistically bounded.

\section{Switched Maps Defined by $C_{\rho k}$ and $C_{k}$ Classes of Primary Self-Mappings and a Class of Dynamic Systems}

Switching processes are a very important tool in some applications of discrete-time and continuous-time dynamic systems. The basic idea is how to switch in-between alternative parameterizations of a system by using either "ad hoc" or even arbitrary switching laws while keeping or improving essential suitable properties like global or asymptotic stability or convergence to the equilibrium points. See [23-26] and some references therein. The formalism can also rely on the definitions of iteration-dependent maps in iterative schemes of Mann or Jungck type or its generalizations so as to get appropriate convergence properties $[18,19,23]$. Note that a switching process in an iterative scheme can be interpreted as the choice under a switching rule of certain primary selfmaps from an available collection of them at certain iteration points; that is, the iterative scheme or the solution equation of a dynamic system is being governed by a switching rule [28]. Based on the above elementary idea, this section relies on switching maps built with a prefixed number of either $C_{\rho k}$-class or $C_{k}$-class, self-mappings on PM-spaces subject to switching rules which select the new selected mapping and the points at which such new switching occurs. For exposition simplicity, it is assumed that $C_{\rho k}$-class, or $C_{k^{-}}$ class, self-mappings are characterized by constants instead of functions in Definitions 1 and 2.

Let $(X, \mathbf{F})$ be PM-space and let $T_{i}: X \rightarrow X$ be a set of (primary) self-mappings of $C_{\rho k}$-class for some constants $\rho_{i}\left(\leq k_{i}\right), k_{i} \in \mathbf{R}_{+}$for $i \in \bar{q}=\{1,2, \ldots, q\}$. A switching map $T=T_{\sigma_{n}}(x)$ from $\mathbf{Z}_{0+} \times X$ to $X$ with respect to the switching law $\sigma: \mathbf{Z}_{0+} \times X \rightarrow \bar{q}$ generates a sequence

$$
x_{n+1}=T x_{n}=T_{\sigma_{n}}\left(x_{n}\right) x_{n}=T_{i} x_{n}, \quad \forall n \in \mathbf{Z}_{0+},
$$

for each given $x_{0} \in X$ for some $i=i(n) \in \bar{q}$ and we informally can say that the $i$ th primary self-mapping $T_{i}: X \rightarrow X$ is "active" at the $n$th value (or sample) of the sequence $\left\{x_{n}\right\}$ [28].
See also [23-27]. In other words, the switching map $T=T_{\sigma_{n}}$ on $X$ is defined by one of the self-mappings $T_{i}: X \rightarrow \stackrel{\sigma_{n}}{X}$ $\left(i \in \bar{q}\right.$ ) for each $n \in \mathbf{Z}_{0+}$ and it has associated piecewise constant functions $\rho_{\sigma_{n}}, k_{\sigma_{n}}: \mathbf{Z}_{0+} \times X \times X \rightarrow \mathbf{R}_{+}$such that $\rho=$ $\rho_{\sigma_{n}}(x, y) \in\left\{\rho_{1}, \rho_{2}, \ldots, \rho_{q}\right\}, k=k_{\sigma_{n}}(x, y) \in\left\{k_{1}, k_{2}, \ldots, k_{q}\right\}$ for each $n \in \mathbf{Z}_{0+}$ and each $x, y \in X$. The set of switching samples of a sequence $\left\{x_{n}\right\} \subset X$ is a (proper or improper) subset $Z_{S}=Z_{S}\left(\left\{x_{n}\right\}, \sigma\right)$ of $\mathbf{Z}_{0+}$, so-called the switching set, defined by $Z_{S}=\left\{n \in \mathbf{Z}_{+}: T_{\sigma_{n}} x_{n} \neq T_{\sigma_{n-1}} x_{n}\right\}=\left\{n_{0}, n_{1}, \ldots, n_{k}, \ldots\right\}$. Note that a switching set is a strictly ordered set for the standard strict ordering relation " $<$ ". Since $T_{i}: X \rightarrow X$, $\forall i \in \bar{q}$, are self-mappings of $C_{\rho k}$ with constants $\rho_{i}\left(\leq k_{i}\right)$, $k_{i} \in \mathbf{R}_{+}$, then

$$
F_{T_{i} x, x}\left(\rho_{i}^{-1} t\right) \geq F_{T_{i}^{2} x, T_{i} x}(t) \geq F_{T_{i} x, x}\left(k_{i}^{-1} t\right) ; \quad \forall t \in \mathbf{R}_{+},
$$

for any $x \in X$ so that one has recursively from (3) for a sequence $\left\{x_{n}\right\} \subset X$ generated by $x_{n+1}=T_{\sigma_{n}} x_{n}, \forall n \in \mathbf{Z}_{0+}$, for any given $x_{0} \in X$

$$
\begin{array}{r}
F_{x_{1}, x_{0}}\left(\left(\prod_{i=0}^{n}\left[\rho_{\sigma_{i}}^{-1}\right]\right) t\right) \\
=F_{x_{1}, x_{0}}\left(\left(\prod_{i=1}^{q}\left[\rho_{i}^{-\sum_{j=0}^{k-1} \gamma_{i}\left(n_{j}, n_{j+1}\right)-\gamma_{i}\left(n_{k}, n_{k}+\ell\right)}\right]\right) t\right) \\
\geq F_{x_{n_{k}+\ell+1}, x_{n_{k}+\ell}}(t) \geq F_{x_{1}, x_{0}}\left(\left(\prod_{i=0}^{n_{k}+\ell}\left[k_{\sigma_{i}}^{-1}\right]\right) t\right) \\
=F_{x_{1}, x_{0}}\left(\left(\prod_{i=1}^{q}\left[k_{i}^{-\sum_{j=0}^{k-1} \gamma_{i}\left(n_{j}, n_{j+1}\right)-\gamma_{i}\left(n_{k}, n_{k}+\ell\right)}\right]\right) t\right) ; \\
\forall k \in \mathbf{Z}_{0+}, \forall t \in \mathbf{R}_{+},
\end{array}
$$

where $\ell\left(\in \mathbf{Z}_{0+}\right) \leq n_{j+1}-n_{j}, n_{k} \in Z_{S}, \forall k \in \mathbf{Z}_{0+}$, is

$$
n_{k}=n_{k-1}+\sum_{i=1}^{q} \gamma_{i}\left(n_{k-1}, n_{k}\right)=\sum_{j=0}^{k-1} \sum_{i=1}^{q} \gamma_{i}\left(n_{j}, n_{j+1}\right)
$$

and $\gamma_{i}\left(n_{j}, n_{j}+\ell\right) \in \mathbf{Z}_{0+}$ is the number of times that the $i$ th self-mapping $T_{i}: X \rightarrow X$ for some $i \in \bar{q}$ is "active" in the interval $\left[n_{j}, n_{j}+\ell\right)$ for each $i \in \bar{q}$. If $T_{i}: X \rightarrow X, \forall i \in \bar{q}$, are self-mappings of $C_{k}$-class, then one has instead of (25)

$$
\begin{array}{r}
F_{x_{n_{k}+\ell+1}, x_{n_{k}+\ell}}(t) \\
\geq F_{x_{1}, x_{0}}\left(\left(\prod_{i=1}^{q}\left[k_{i}^{-\sum_{j=0}^{k-1} \gamma_{i}\left(n_{j}, n_{j+1}\right)-\gamma_{i}\left(n_{k}, n_{k}+\ell\right)}\right]\right) t\right) ; \\
\forall k \in \mathbf{Z}_{0+}, \forall t \in \mathbf{R}_{+} .
\end{array}
$$

Theorem 14. Let $(X, \mathbf{F})$ be a PM-space and let $T_{i}: X \rightarrow X$ be self-mappings of $C_{k}$-class for some constants $k_{i} \in \mathbf{R}_{+}$for $i \in \bar{q}=\{1,2, \ldots, q\}$. Then, the following properties hold:

(i) Assume that there is (at least) a self-mapping $T_{i}$ : $X \rightarrow X$ for some $i \in \bar{q}$ which is a probabilistic strict contraction. Then, there are infinitely many switching laws $\sigma: \mathbf{Z}_{0+} \times X \rightarrow \bar{q}$ such that their associate switching maps $T: \mathbf{Z}_{0+} \times X \rightarrow X$ are probabilistic strict contractions. 
(ii) Under the conditions of the above proposition, there are infinitely many switching laws $\sigma: \mathbf{Z}_{0+} \times X \rightarrow \bar{q}$ such that their associate switching maps $T: \mathbf{Z}_{0+} \times X \rightarrow X$ are probabilistic strict contractions and, furthermore, they consist of infinitely many alternate active switching maps of the form $(i, j)$ or $(j, i)$ with $j \in \bar{q} \backslash\{i\}$.

(iii) If, in addition, $(X, \mathbf{F})$ is complete, then any sequence $x_{n+1}=T_{\sigma_{n}} x_{n}, n \in \mathbf{Z}_{0+}$ under any switching law $\sigma$ : $\mathbf{Z}_{0+} \times X \rightarrow \bar{q}$ fulfilling either Property (i) or Property (ii) for any given initial point $x_{0} \in X$ is Cauchy and probabilistically convergent.

Proof. It follows from (27) that Property (i) is fulfilled for sequences $\left\{x_{n}\right\} \subset X$ generated as $x_{n+1}=T_{\sigma_{n}} x_{n}$ for any $x_{0} \in X$ by any of the infinitely many switching maps $T: \mathbf{Z}_{0+} \times X \rightarrow$ $X$ built under switching laws $\sigma: \mathbf{Z}_{0+} \times X \rightarrow \bar{q}$ which fulfil

$$
\begin{aligned}
& \lim _{k \rightarrow \infty} \sum_{j=0}^{k-1} \gamma_{i}\left(n_{j}, n_{j+1}\right)=+\infty, \\
& \sum_{\ell(\neq i)=1}^{q} \sum_{k=0}^{\infty} \gamma_{\ell}\left(n_{k}, n_{k+1}\right)<+\infty
\end{aligned}
$$

since there is a finite nonnegative integer $n^{*}=n^{*}(\sigma)$ depending on the subsequence $\left\{x_{n}: n<n^{*}\right\}$ which is a terminal switching point such that $\sigma_{n}=i$ for $i \geq n^{*}$ for any such a sequence $\left\{x_{n}\right\}$. Thus, one gets from (27) that

$$
\lim _{n \rightarrow \infty} F_{x_{n+m}, x_{n}}(t)=F_{T_{\sigma_{0}} x_{0}, x_{0}}(+\infty)=1,
$$

$$
\forall t \in \mathbf{R}_{+}, \forall m \in \mathbf{Z}_{+} \text {, }
$$

since $F: \mathbf{R} \rightarrow \mathbf{R}_{0+}$ is nondecreasing and left-continuous with $\sup _{t \in \mathbf{R}} F(t)=1$ and then the sequence $\left\{x_{n}\right\}$ built as $x_{n+1}=T x_{n}=T_{\sigma_{n}} x_{n}, x_{0} \in X$ is a Cauchy sequence and $T: \mathbf{Z}_{0+} \times X \rightarrow X$ is a probabilistic strict contraction. Property (i) has been proved.

Property (ii) follows with alternate (probabilistic strict contraction versus remaining self-mapping) switching laws $\sigma: \mathbf{Z}_{0+} \times X \rightarrow \bar{q}$ defined by a switching set $Z_{S}$ fulfilling the fact that if, for any $k \in \mathbf{Z}_{0+}, n_{k+j} \in Z_{S}$ for $j=0,1, \ldots, \ell_{k}-2$ has active (perhaps nonprobabilistic strict contractions) selfmappings $T_{j}: X \rightarrow X$ for $j(\neq i) \in \bar{q}$, then $n_{k+\ell_{k}} \in Z_{S}$ is defined such that $T_{i}: X \rightarrow X$ is active on $\left[n_{k+\ell_{k}-1}, n_{k+\ell_{k}}\right]$; that is, $x_{n+1}=T_{\sigma_{n}} x_{n}=T_{i} x_{n}$ for $n \in\left\{n_{k+\ell_{k}-1}, n_{k+\ell_{k}}\right\}$ with $n_{k+\ell_{k}}$ being defined with $\ell_{k}$ large enough such that

$$
k_{i}^{n_{k+\ell_{k}}-n_{k+\ell_{k}-1}}<\prod_{j(\neq i)=1}^{q}\left[k_{j}^{\sum_{\ell=k}^{k+\ell_{k}-1} \gamma_{j}\left(n_{\ell}, n_{\ell+1}\right)}\right]
$$

which lead to

$$
\begin{aligned}
\lim _{k \rightarrow \infty} \sum_{j=0}^{k-1} \gamma_{i}\left(n_{j}, n_{j+1}\right) & =\lim _{k \rightarrow \infty} \sum_{\ell(\neq i)=1}^{q} \sum_{k=0}^{\infty} \gamma_{\ell}\left(n_{k}, n_{k+1}\right) \\
& =+\infty
\end{aligned}
$$

so that we get again (29) and a similar conclusion. Property (iii) is obvious from the fact that $(X, \mathbf{F})$ is complete and $\left\{x_{n}\right\}$ is Cauchy under switching laws fulfilling either Property (i) or Property (ii).

The following result is a direct consequence of Theorem 14 since mappings of $C_{\rho k}$-class are also of $C_{k^{-}}$ class.

Corollary 15. Let $(X, \mathbf{F})$ be a PM-space and let $T_{i}: X \rightarrow X$ be self-mappings of $C_{\rho k}$-class for some constants $\rho_{i}\left(\leq k_{i}\right), k_{i} \in$ $\mathbf{R}_{+}$, for $i \in \bar{q}=\{1,2, \ldots, q\}$. Then, Theorem 14 still holds.

Theorem 16. Let $(X, \mathrm{~F}, \Delta)$ be a complete Menger space with $\Delta(a, b)=\min (a, b)$ and let $T_{j}: X \rightarrow X$ be self-mappings of $C_{k}$-class for some constants $k_{j} \in \mathbf{R}_{+}$for all $j \in \bar{q}=$ $\{1,2, \ldots, q\}$ with at least $T_{i}: X \rightarrow X$ being a probabilistic strict contraction for some $i \in \bar{q}$. Let $\sigma: \mathbf{Z}_{0+} \times X \rightarrow \bar{q}$ be a switching law and let $\left\{x_{n}\right\} \subset X$ be a sequence generated as $x_{n+1}=T_{\sigma_{n}} x_{n}, n \in \mathbf{Z}_{0+}$, for any given $x_{0} \in X$ such that their associate switching map $T: \mathbf{Z}_{0+} \times X \rightarrow X$ is defined by $T x_{n}=T_{\sigma_{n}} x_{n}=T_{i} x_{n}$ for $j \in \bar{q}$ and all $n \geq n^{*}$ and some finite $n^{*} \in \mathbf{Z}_{0+}$. Then, $\left\{x_{n}\right\} \rightarrow z_{i}$ which is the unique fixed point of the strict contraction $T_{i}: X \rightarrow X$.

Proof. Since $T_{i}: X \rightarrow X$ is a strict probabilistic contraction, $\lim _{n \rightarrow \infty} F_{x_{n+m}, x_{n}}(t)=F_{T_{i} x_{n^{*}}, x_{n^{*}}}(+\infty)=1, \forall t \in \mathbf{R}_{+}, \forall m \in \mathbf{Z}_{+}$, from (29) since $T x_{n}=T_{\sigma_{n}} x_{n}=T_{i} x_{n}$ for $n \geq n^{*}$. Then $\left\{x_{n}\right\}$ is probabilistically convergent to $z_{i} \in X$ which is a fixed point of the probabilistic strict contraction $T_{i}: X \rightarrow X$ as proved by contradiction. Assume that this is false so that $z_{i} \neq T_{i} z_{i}$ and then since $\left\{x_{n}\right\}$ is Cauchy, $\left\{x_{n}\right\} \rightarrow z_{i}, F: \mathbf{R} \rightarrow \mathbf{R}_{0+}$ which is nondecreasing and left-continuous, and $T_{i}: X \rightarrow X$ is a probabilistic strict contraction, one gets, for any given $t \in \mathbf{R}_{+}$ and $\lambda \in(0,1)$ and all $n\left(\in \mathbf{Z}_{0+}\right) \geq N$ and some $N=N(t, \lambda) \in$ $\mathbf{Z}_{0+}$,

$$
\begin{aligned}
1- & \lambda_{0}(t) \geq F_{z_{i}, T_{i} z_{i}}(t) \\
& \geq \Delta\left(\Delta\left(F_{z_{i}, x_{n}}\left(\frac{t}{4}\right), F_{T_{i} x_{n}, x_{n}}\left(\frac{t}{4}\right)\right), F_{T_{i} x_{n}, T_{i} z_{i}}\left(\frac{t}{2}\right)\right) \\
& \geq \Delta\left(\Delta\left(F_{z_{i}, x_{n}}\left(\frac{t}{4}\right), F_{T_{i} x_{n}, x_{n}}\left(\frac{t}{4}\right)\right), F_{T_{i} x_{n}, T z_{i}}\left(\frac{t}{4}\right)\right) \\
& \geq \Delta\left(\Delta\left(F_{z_{i}, x_{n}}\left(\frac{t}{4}\right), F_{T_{i} x_{n}, x_{n}}\left(\frac{t}{4}\right)\right), F_{x_{n}, z_{i}}\left(k_{i}^{-1} \frac{t}{4}\right)\right) \\
& \geq \Delta\left(\Delta\left(F_{z_{i}, x_{n}}\left(\frac{t}{4}\right), F_{T_{i} x_{n}, x_{n}}\left(\frac{t}{4}\right)\right), F_{x_{n}, z_{i}}\left(\frac{t}{4}\right)\right) \\
& >1-\lambda
\end{aligned}
$$

for some $\lambda_{0}=\lambda_{0}(t)>0, \forall t \in \mathbf{R}_{+}$, which implies that $\lambda \epsilon$ $\left(\lambda_{0}, 1\right)$ but since $\lambda \in(0,1)$ can be chosen arbitrarily, it suffices to take $\lambda \in\left(0, \lambda_{0}\right]$ to get a contradiction. Then, $z_{i}=T z_{i}$ which is proved to be unique again by contradiction. Assume that this is not the case so that there exist $z_{i 1}=T^{n} z_{i 1}$ and $z_{i 2}=T^{n} z_{i 2} \neq z_{i 1}, \forall n \in \mathbf{Z}_{0+}$, which are fixed points of the probabilistic strict contraction $T_{i}: X \rightarrow X$. Thus, one gets the contradiction

$$
\begin{aligned}
1 & >F_{z_{i 1}, z_{i 2}}\left(0^{+}\right)=F_{z_{i 1}, z_{i 2}}(t)=F_{T^{n} z_{i 1}, T^{n} z_{i 2}}(t) \\
& \geq F_{z_{i 1}, z_{i 2}}\left(k_{i}^{-n} t\right)=F_{z_{i 1}, z_{i 2}}(+\infty)=1,
\end{aligned}
$$


so that $z_{i 2}=z_{i 1}=z_{i}=T_{i} z_{i}$. Since $T x_{n}=T_{\sigma_{n}} x_{n}=T_{i} x_{n}$ for $n \geq n^{*}$ for some finite $n^{*} \in \mathbf{Z}_{0+}$, we can write $x_{n^{*}}=T_{\sigma_{n^{*}-1}} \ldots \ldots$ $T_{\sigma_{0}} x_{0}$ and $x_{n}=T_{i}^{n-n^{*}} x_{n^{*}}$ for $n\left(\in \mathbf{Z}_{0+}\right) \geq n^{*}$ to get that $\left\{x_{n}\right\}$ generated by $x_{n+1}=T_{\sigma_{n}} x_{n}, \forall n \in \mathbf{Z}_{0+}$, for any given arbitrary $x_{0} \in X$ is probabilistically convergent to $z_{i}=T_{i} z_{i}$.

The above result is a direct consequence of Theorem 14 which is also valid if $T_{i}: X \rightarrow X$ is of $C_{\rho k}$-class. However, note that, under the alternate switching laws in Theorem 14(ii), the limit points of sequences generated through the switching maps $T: \mathbf{Z}_{0+} \times X \rightarrow X$ are, in general, dependent on the initial points of the sequences and on the switching law.

The following result generalizes Theorem 16 without assuming any special contractive condition on at least one of $T_{i}: X \rightarrow X, \forall i \in \bar{q}$, with the only condition on the operators being that all of them are either of $C_{\rho k}$-class or $C_{k}$-class.

Theorem 17. Let $(X, \mathbf{F})$ be a PM-space, let $T_{i}: X \rightarrow X$ be self-mappings of $C_{\rho k}$-class for some constants $\rho_{i}\left(\leq k_{i}\right), k_{i} \in \mathbf{R}_{+}$, $\forall i \in \bar{q}$, and let $T: \mathbf{Z}_{0+} \times X \rightarrow X$ be a switching mapping associated with a switching law $\sigma: \mathbf{Z}_{0+} \times X \rightarrow \bar{q}$ which generates a sequence $\left\{x_{n}\right\} \subset X$ as $x_{n+1}=T_{\sigma_{n}} x_{n}, \forall n \in \mathbf{Z}_{0+}$, for some given $x_{0} \in X$. Let $Z_{S} \subseteq Z_{0+}$ be the set of switching points, that is, for any given $n_{k} \in Z_{S}, \forall k \in \mathbf{Z}_{+}$, provided that $n_{k-1} \in Z_{S}$, if and only if $\sigma_{n_{k}} \neq \sigma_{n_{k}-1}=\sigma_{n_{k-1}}$. Then, the following properties hold:

(i)

$$
F_{T x_{0}, x_{0}}\left(\bar{\rho}_{n} t\right) \geq F_{x_{n+1}, x_{n}}(t) \geq F_{T x_{0}, x_{0}}\left(\bar{\gamma}_{n} t\right) ; \quad \forall t \in \mathbf{R}_{+},
$$

for all $n \in\left[n_{k}, n_{k}+\ell\right) \cap \mathbf{Z}_{0+}, \ell\left(\in \mathbf{Z}_{0+}\right) \leq n_{k+1}-n_{k}$, $n_{k} \in Z_{S}, \forall k \in \mathbf{Z}_{0+}$, where

$$
\begin{aligned}
& \bar{\gamma}_{n}=\prod_{i=1}^{q}\left[k_{i}^{-\sum_{j=0}^{k-1} \gamma_{i}\left(n_{j}, n_{j+1}\right)-\gamma_{i}\left(n_{k}, n_{k}+\ell\right)}\right], \\
& \bar{\rho}_{n}=\prod_{i=1}^{q}\left[\rho_{i}^{-\sum_{j=0}^{k-1} \gamma_{i}\left(n_{j}, n_{j+1}\right)-\gamma_{i}\left(n_{k}, n_{k}+\ell\right)}\right],
\end{aligned}
$$

where $\gamma_{i}\left(n_{j}, n_{j}+\ell\right) \in \mathbf{Z}_{0+}$ is the number of times that the ith self-mapping $T_{i}: X \rightarrow X$ for each $i \in \bar{q}$ is "active" in the interval $\left[n_{j}, n_{j}+\ell\right)$ for some $i \in \bar{q}$.

(ii) If $F: \mathbf{R} \rightarrow[0,1]$ is upper-semicontinuous at $\bar{\rho} t$ for some given $t \in \mathbf{R}_{+}$, where $\bar{\rho}=\limsup _{n \rightarrow \infty} \bar{\rho}_{n}$, then $\left\{x_{n}\right\}$ has the following property:

$$
\limsup _{n \rightarrow \infty} F_{x_{n+1}, x_{n}}(t) \leq F_{T x_{0}, x_{0}}(\bar{\rho} t) .
$$

If $F: \mathbf{R} \rightarrow[0,1]$ is lower-semicontinuous at $\bar{\rho} t$ for some given $t \in \mathbf{R}_{+}$, where $\gamma=\liminf _{n \rightarrow \infty} \bar{\gamma}_{n}$, then $\left\{x_{n}\right\}$ has the following property:

$$
\liminf _{n \rightarrow \infty} F_{x_{n+1}, x_{n}}(t) \geq F_{T x_{0}, x_{0}}(\underline{\gamma} t) .
$$

(iii) If $T_{i}: X \rightarrow X$ are of $C_{k}$-class for some constants $k_{i} \epsilon$ $\mathbf{R}_{+}, \forall i \in \bar{q}$, then $\left\{x_{n}\right\}$ has the following property:

$$
F_{x_{n+1}, x_{n}}(t) \geq F_{T x_{0}, x_{0}}\left(\bar{\gamma}_{n} t\right) ; \quad \forall t \in \mathbf{R}_{+} .
$$

And all $n \in\left[n_{k}, n_{k}+\ell\right) \cap \mathbf{Z}_{0+}, \ell\left(\in \mathbf{Z}_{0+}\right) \leq n_{k+1}-$ $n_{k}, n_{k} \in Z_{S}, \forall k \in \mathbf{Z}_{0+}$. If $F: \mathbf{R} \rightarrow[0,1]$ is lowersemicontinuous at $\bar{\rho}$ for a given $t \in \mathbf{R}_{+}$, where $\underline{\gamma}=$ $\liminf _{n \rightarrow \infty} \bar{\gamma}_{n}$, then (38) holds.

Proof. Property (i) follows since (34), subject to (35)-(36), is obtained directly from (25)-(26). If $F: \mathbf{R} \rightarrow[0,1]$ is uppersemicontinuous at $\bar{\rho} t$, then

$$
\begin{aligned}
\limsup _{n \rightarrow \infty} F_{x_{n+1}, x_{n}}(t) & \leq \limsup _{\bar{\rho}_{n} \rightarrow \bar{\rho}} F_{T x_{0}, x_{0}}\left(\bar{\rho}_{n} t\right) \\
& \leq F_{T x_{0}, x_{0}}\left(\left(\limsup _{\bar{\rho}_{n} \rightarrow \bar{\rho}} \bar{\rho}_{n}\right) t\right) \\
& =F_{T x_{0}, x_{0}}(\bar{\rho} t) .
\end{aligned}
$$

In the same way, if $F: \mathbf{R} \rightarrow[0,1]$ is lower-semicontinuous at $\gamma t$, we get $\liminf _{n \rightarrow \infty} F_{x_{n+1}, x_{n}}(t) \geq F_{T x_{0}, x_{0}}(\gamma t)=$ $F_{T x_{0}, x_{0}}\left(\left(\lim \inf _{\bar{\gamma}_{n} \rightarrow \gamma} \bar{\gamma}_{n}\right) t\right)$. This proves Property (ii). Property (iii) is a restriction of Properties (i)-(ii) for the case when $T_{i}: X \rightarrow X$ are of $C_{k}$-class for some constants $k_{i} \in \mathbf{R}_{+}$, $\forall i \in \bar{q}$.

Note that although $F: \mathbf{R} \rightarrow[0,1]$ is assumed to be everywhere left-continuous in the probabilistic metric framework, this does not mean that it is everywhere lower- and/or uppersemicontinuous. Therefore, some extra related conditions are imposed in Theorem 17(ii)-(iii) allowing obtaining limit upper- and lower-bounds of $F_{x_{n+1}, x_{n}}(t)$ as $n \rightarrow \infty$ via the limit superior and the limit inferior.

The following two results related to bounded and unbounded sequences follow from Theorem 17.

Corollary 18. Let $(X, \mathbf{F})$ be a PM-space, let $T_{i}: X \rightarrow X$ be self-mappings of $C_{k}$-class for some constants $k_{i} \in \mathbf{R}_{+}, \forall i \in \bar{q}$, and let $T: Z_{0+} \times X \rightarrow X$ be a switching mapping associated with a switching law $\sigma: \mathbf{Z}_{0+} \times X \rightarrow \bar{q}$ which generates $a$ sequence $\left\{x_{n}\right\} \subset X$ as $x_{n+1}=T_{\sigma_{n}} x_{n}, \forall n \in \mathbf{Z}_{0+}, \forall n \in \mathbf{Z}_{0+}$, for some given $x_{0} \in X$ with switching points in the switching set $Z_{S}$. Assume also that $F: \mathbf{R} \rightarrow[0,1]$ is everywhere lowersemicontinuous with $\gamma=\liminf _{n \rightarrow \infty} \bar{\gamma}_{n}>0$ for such a sequence $\left\{x_{n}\right\}$. Then, the following properties hold:

(i) $\left\{x_{n}\right\}$ is probabilistically bounded.

(ii) If $F_{x_{0}, T x_{0}}(t)=H(t-d(x, T x)), \forall x_{0} \in X, \forall t \in \mathbf{R}$, then $\exists \lim _{n \rightarrow \infty} H\left(t-d\left(T^{n+1} x_{0}, T^{n} x_{0}\right)\right)=1$ if $t \in$ $\left(d\left(x_{0}, T x_{0}\right) / \gamma,+\infty\right)$.

Proof. From (39), one concludes (38); that is, $\liminf _{n \rightarrow \infty} F_{x_{n+1}, x_{n}}(t) \geq F_{T x_{0}, x_{0}}(\gamma t), \forall t \in \mathbf{R}_{+}$. Since $\gamma>0$, then $\sup _{t \in \mathbf{R}_{0+}} F_{T x_{0}, x_{0}}(\gamma t)=1$ and $\left\{x_{n}\right\}$ is probabilistically bounded since a nonempty set $A=A\left(\left\{x_{n_{k}}\right\}\right) \subset X$ which contains all the points of some subsequence $\left\{x_{n_{k}}\right\} \subseteq\left\{x_{n}\right\}$ has the property that $D_{A} \in D_{+}$. Property (i) has been proved. 
On the other hand, it follows from (25) and Property (i) that if $F_{x_{0}, T x_{0}}(t)=H(t-d(x, T x)), \forall x_{0} \in X, \forall t \in \mathbf{R}$, then

$$
\begin{aligned}
& \lim _{n \rightarrow \infty} H\left(t-d\left(T^{n+1} x_{0}, T^{n} x_{0}\right)\right) \\
& \geq H\left(\underline{\gamma t}-d\left(x_{0}, T x_{0}\right)\right)=1 ; \\
& \quad t \in\left(\frac{d\left(x_{0}, T x_{0}\right)}{\underline{\gamma}},+\infty\right)
\end{aligned}
$$

and Property (ii) is proved.

Since mappings of $C_{k}$-class are also of $C_{\rho k}$-class, then Corollary 18 also holds if some of $T_{i}: X \rightarrow X$ are selfmappings of $C_{\rho k}$-class for some constants $\rho_{i}\left(\leq k_{i}\right), k_{i} \in \mathbf{R}_{+}$, $\forall i \in \bar{q}$.

Corollary 19. Let $(X, \mathbf{F})$ be a PM-space, let $T_{i}: X \rightarrow X$ be self-mappings of $C_{k}$-class for some constants $k_{i} \in \mathbf{R}_{+}, \forall i \in \bar{q}$, and let $T: \mathbf{Z}_{0+} \times X \rightarrow X$ be a switching mapping associated with a switching law $\sigma: \mathbf{Z}_{0+} \times X \rightarrow \bar{q}$ which generates $a$ sequence $\left\{x_{n}\right\} \subset X$ as $x_{n+1}=T_{\sigma_{n}} x_{n}, \forall n \in \mathbf{Z}_{0+}, \forall n \in \mathbf{Z}_{0+}$, for some given $x_{0} \in X$ with switching points in the switching set $Z_{S}$. Assume also that $F: \mathbf{R} \rightarrow[0,1]$ is everywhere uppersemicontinuous with $\bar{\rho}=\limsup _{n \rightarrow \infty} \bar{\rho}_{n}=0$ for such $a$ sequence $\left\{x_{n}\right\}$. Then, the following properties hold:

(i) $\left\{x_{n}\right\}$ is probabilistically unbounded.

(ii) If $F_{x_{0}, T x_{0}}(t)=H(t-d(x, T x)), \forall x_{0} \in X, \forall t \in \mathbf{R}$, then $\exists \lim _{t \rightarrow+\infty} \lim _{n \rightarrow \infty} H\left(t-d\left(T^{n+1} x_{0}, T^{n} x_{0}\right)\right)=1$.

Proof. From (39), one concludes (38); that is, $\limsup _{n \rightarrow \infty} F_{x_{n+1}, x_{n}}(t) \leq F_{T x_{0}, x_{0}}(\bar{\rho} t)=F_{T x_{0}, x_{0}}(0)=0$, $\forall t \in \mathbf{R}_{+}$. Consider a nonempty set $A=A\left(\left\{x_{n_{k}}\right\}\right) \subset X$ which contains all the points of some subsequence $\left\{x_{n_{k}}\right\} \subseteq\left\{x_{n}\right\}$. It is obvious that $D_{A} \notin D_{+}$. Then $\left\{x_{n}\right\}$ is probabilistically unbounded since it has a probabilistically unbounded subsequence. Property (i) has been proved. On the other hand, it follows from (25) and Property (i) that if $F_{x_{0}, T x_{0}}(t)=H(t-d(x, T x)), \forall x_{0} \in X, \forall t \in \mathbf{R}$, then

$$
\begin{aligned}
& \lim _{t \rightarrow+\infty} \lim _{n \rightarrow \infty} H\left(t-d\left(T^{n+1} x_{0}, T^{n} x_{0}\right)\right) \\
& \quad \leq \lim _{t \rightarrow+\infty} H\left(\bar{\rho} t-d\left(x_{0}, T x_{0}\right)\right)=H\left(-d\left(x_{0}, T x_{0}\right)\right) \\
& \quad=H(0)=0
\end{aligned}
$$

and Property (ii) is proved.

Remarks. (1) Note that Corollary 18 is fulfilled, in particular, by switched sequences with switching set of finite cardinal with a terminal point of switching to some nonexpansive $C_{\rho k}$ (or $C_{k}$ ) self-mapping $T_{i}: X \rightarrow X$; that is, $k_{i} \in(0,1]$ for some $i \in \bar{q}$. Note also that Corollary 18 is not fulfilled for terminal switching to an expansive self-mapping.

(2) Note that Corollary 19 for $C_{\rho k}$ self-mappings can also be applied to self-mappings $T_{i}: X \rightarrow X, i \in \bar{q}$, which are only subject to the upper-bounding rule of the probability density function (say self-mappings "of $C_{\rho}$-class"); that is,

$$
F_{T_{i} x_{0}, x_{0}}\left(\rho_{i}^{-1} t\right) \geq F_{T_{i} x_{0}, T_{i}^{2} x_{0}}(t) ; \quad \forall t \in \mathbf{R}_{+}
$$

while leading to a similar conclusion about probabilistic unboundedness. Note that the result applies, in particular, to switched sequences with switching set of finite cardinal with a terminal point of switching to some expansive $C_{\rho k}$ (or $C_{\rho}$ ) self-mapping $T_{i}: X \rightarrow X$ for some $i \in \bar{q}$; that is, $\rho_{i}>1$ for some $i \in \bar{q}$.

(3) Note that Corollary 18 is also fulfilled by switched sequences with switching set of infinite cardinal if $\gamma>0$. Corollary 18 excludes switching laws $\sigma: \mathbf{Z}_{0+} \times X \rightarrow \overline{\bar{q}}$ being built under some expansive self-mapping $T_{i}: X \rightarrow X$ for some $i \in \bar{q}$ being used so infinitely often such that $\gamma=0$ with either finite terminal switching point or not. This includes, in particular, the case when the switched map is built with a finite terminal switching point to an expansive self-mapping. Then, the probabilistic unboundedness result of Corollary 19 applies.

Note that an expansive mapping can give a fixed point which unbounded sequences do not converge to as the next example visualizes.

Example 20. Assume that $(X, \mathbf{F})$ is a PM-space with $0 \in X$ and consider a self-map $T: X \rightarrow X$ such that $T 0=0$. Thus, $z=0$ is trivially a fixed point of $T: X \rightarrow X$. Assume the following cases:

(a) $F_{0, x}(t) \geq F_{0, T x}(\rho t), \forall t \in \mathbf{R}_{+}$, and all $x(\neq 0) \in X$ for some real constant $\rho>1$ so that $T: X \rightarrow X$ is expansive. Then, $F_{0, T^{n}{ }_{x}}(t) \leq F_{0, x}\left(\rho^{-n} t\right), \forall t \in \mathbf{R}_{+}$, $\forall n \in \mathbf{Z}_{0+}$, so that $\lim _{n \rightarrow \infty} F_{0, T^{n} x}(t)=0, \forall t \in \mathbf{R}_{+}, \forall n \in$ $\mathbf{Z}_{0+}$, and the sequence $\left\{T^{n} x\right\}$ is unbounded and does not converge in probability to the fixed point $z=0$. There is an analogy with the expansive deterministic counterpart examples. For instance, consider a scalar difference equation $x_{n+1}=\rho x_{n}, \forall n \in \mathbf{Z}_{0+}$, with $|\rho|>1$ and $x_{0} \neq 0$. Then, $z=0$ is a fixed point of the self-mapping on $\mathbf{R}$ defining the sequence trajectory solution which is clearly expansive for the metric space $(\mathbf{R}, d)$ with $d$ being any metric, for instance, the Euclidean norm. However, $\left|x_{n}\right| \rightarrow+\infty$ as $n \rightarrow \infty$ so that the unique fixed point is an unstable equilibrium point.

(b) $F_{0, T x}(t) \geq F_{0, x}\left(k^{-1} t\right), \forall t \in \mathbf{R}_{+}$, and all $x(\neq 0) \in X$ for some real constant $k \in(0,1]$ so that $T: X \rightarrow X$ is nonexpansive. Then, if furthermore $k \in(0,1)$, then $T: X \rightarrow X$ is strictly contractive and $F_{0, T^{n} x}(t) \geq$ $F_{0, x}\left(k^{-n} t\right), \forall n \in \mathbf{Z}_{0+}$, so that $\lim _{n \rightarrow \infty} F_{0, T^{n} x}(t)=1$ and $\left\{T^{n} x\right\} \rightarrow z=0$, which is a fixed point, with probability one, is also Cauchy and probabilistically bounded. The deterministic counterpart can be the example $x_{n+1}=k x_{n}$ with $x_{0} \neq 0, \forall n \in \mathbf{Z}_{0+}$, with $|k|<1$ in a metric space $(\mathbf{R}, d)$, where $z=0$ is the unique fixed point (and a globally 
asymptotically stable equilibrium point) of the selfmapping defining the sequence trajectory solution for any initial condition.

(c) In the above case, assuming $k=1$, then, for any $x \in X$, $F_{T^{n+1} x, T^{n} x}(t) \geq F_{x, T x}(t), \forall t \in \mathbf{R}_{+}, \forall n \in \mathbf{Z}_{0+}$. If $T x=$ $x$, then $F_{T^{n+1} x, T^{n} x}(t)=1, T^{n} x=x, \forall n \in \mathbf{Z}_{0+}$; the mapping $T: X \rightarrow X$ defining the solution is not expansive but not contractive and any point is a fixed point. A deterministic counterpart can be visualized with the example $x_{n+1}=x_{n}, \forall n \in \mathbf{Z}_{0+}$, for any $x_{0} \in X$ which has infinitely many fixed points which are also (nonasymptotically) stable equilibrium points.

(d) The above discussion can be directly extended to the case of switching maps built under switching laws with finite terminal switching point to an expansive primary self-mapping or for appropriate switching laws with no terminal switching point in the presence of at least one expansive primary self-mapping.

A worked numerical example follows.

Example 21. This example aims at numerically illustrating the main results stated and proved in Section 3 through Theorem 17 (as a generalization of Theorem 16). For this purpose, consider

$$
F_{x, y}(t)=H(t-d(x, y))= \begin{cases}0, & t \leq d(x, y) \\ 1, & t>d(x, y)\end{cases}
$$

where $d(x, y)$ is the distance induced by the 2-norm, $d(x, y)=\|x-y\|_{2}$. Consider also the switched self-mapping described by the discrete dynamical system $x_{n+1}=T_{\sigma_{n}} x_{n}$ with $T_{\sigma_{n}}=A_{\sigma_{n}}+F_{\sigma_{n}}, A_{\sigma_{n}} \in\left\{A_{1}, A_{2}, A_{3}\right\}$, and $F_{\sigma_{n}} \in\left\{F_{1}, F_{2}, F_{3}\right\}$. This way, $A$ matrices can be regarded as dynamics matrices while $F$ matrices can be understood as perturbation ones. The dynamics matrices are selected in such a way that in conjunction with the perturbation ones the switched map exhibits different characters (contractive, nonexpansive, and expansive) for each $i=1,2,3$ so that the effect of switching can be positively noticed. Thus, the dynamics matrices are given by

$$
\begin{aligned}
& A_{1}=\left(\begin{array}{lll}
0.1 & 0.3 & 0.2 \\
0.1 & 0.2 & 0.2 \\
0.1 & 0.2 & 0.1
\end{array}\right), \\
& A_{2}=\left(\begin{array}{ccc}
0.2 & 0.3 & 0.4 \\
0.425 & 0.2 & 0.3 \\
0.1 & 0.3 & 0.1
\end{array}\right), \\
& A_{3}=\left(\begin{array}{lll}
1.2 & 0.3 & 1.4 \\
0.5 & 0.2 & 1.3 \\
0.1 & 0.3 & 1.1
\end{array}\right),
\end{aligned}
$$

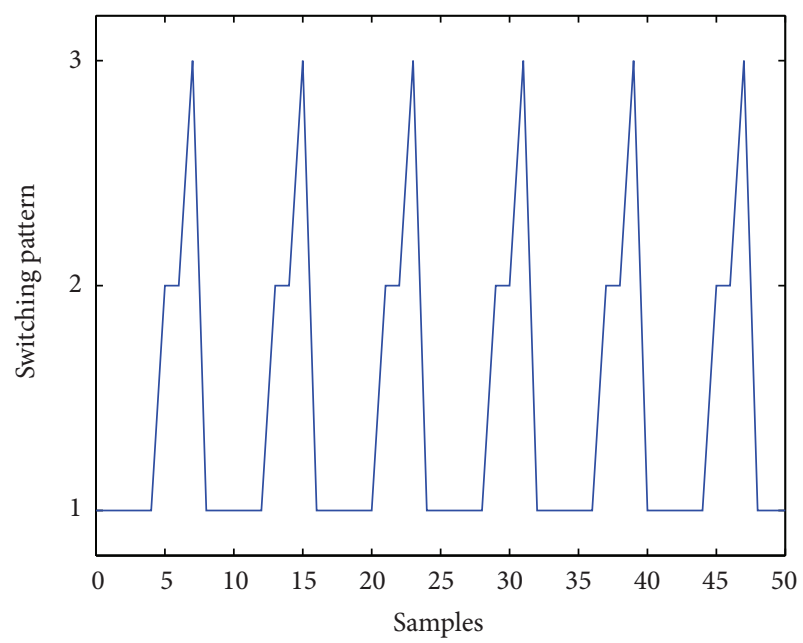

FIGURE 1: Periodic switching map for the first experiment.

whose eigenvalues are, respectively, given by

$$
\begin{aligned}
& \operatorname{spec}\left(A_{1}\right)=\{0.741,-0.17+0.153 j,-0.17-0.153 j\}, \\
& \operatorname{spec}\left(A_{2}\right) \\
& \quad=\{0.7722,-0.1361+0.1191 j,-0.1361-0.1191 j\}, \\
& \operatorname{spec}\left(A_{3}\right)=\{1.8691,0.7381,-0.1073\} .
\end{aligned}
$$

The constants characterizing each one of these matrices, interpreted as operators, are calculated from (2) $F_{T x, T y}(t) \geq$ $F_{x, y}\left(k^{-1} t\right)$, which in this particular case takes the form

$$
\begin{aligned}
H\left(t-d\left(A_{i} x, A_{i} y\right)\right) & \geq H\left(k^{-1} t-d(x, y)\right) \\
& =H\left(\frac{t-k d(x, y)}{k}\right)
\end{aligned}
$$

for each one of the matrices, $i=1,2,3$. The latter condition is satisfied if $d\left(A_{i} x, A_{i} y\right) \leq k_{i} d(x, y)$ which results in the considered metric in $\left\|A_{i} x-A_{i} y\right\|_{2} \leq k_{i}\|x-y\|_{2}$. Therefore, for the first matrix, we have

$$
\begin{aligned}
\left\|A_{1} x-A_{1} y\right\|_{2} & \leq\left\|A_{1}\right\|_{2}\|x-y\|_{2}=0.534\|x-y\|_{2} \\
& <0.54\|x-y\|_{2}
\end{aligned}
$$

so that $k_{1}=0.54$. The 2-norms of the remaining matrices are $\left\|A_{2}\right\|_{2}=0.8$ and $\left\|A_{3}\right\|_{2}=2.528$. As it can be seen, $A_{1}$ and $A_{2}$ are contractive operators while $A_{3}$ is expansive. However, the perturbation matrices $F$ will shape the behavior of the operator $T_{\sigma_{n}}=A_{\sigma_{n}}+F_{\sigma_{n}}$ in a different way. To this end, fix 


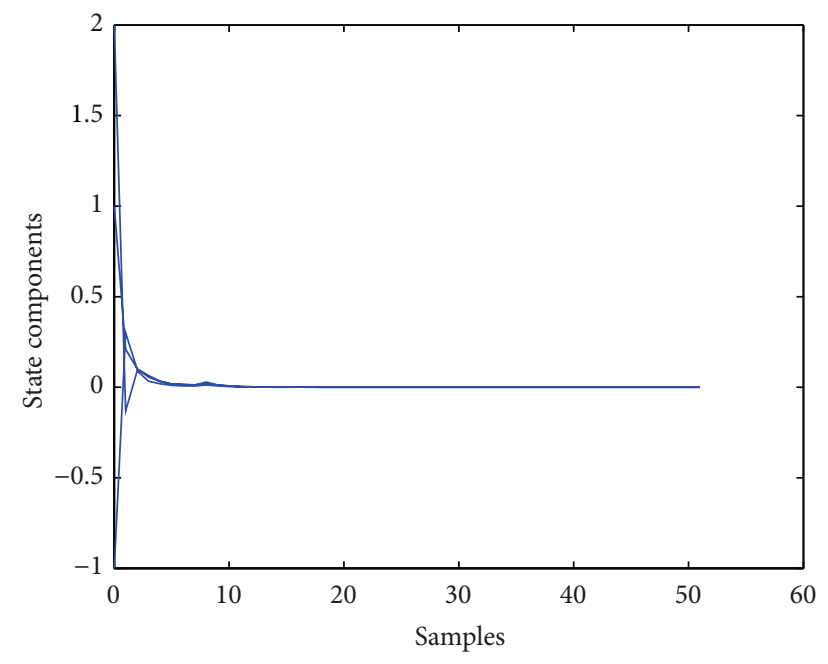

FIgURE 2: Evolution of the state variables for the first experiment.

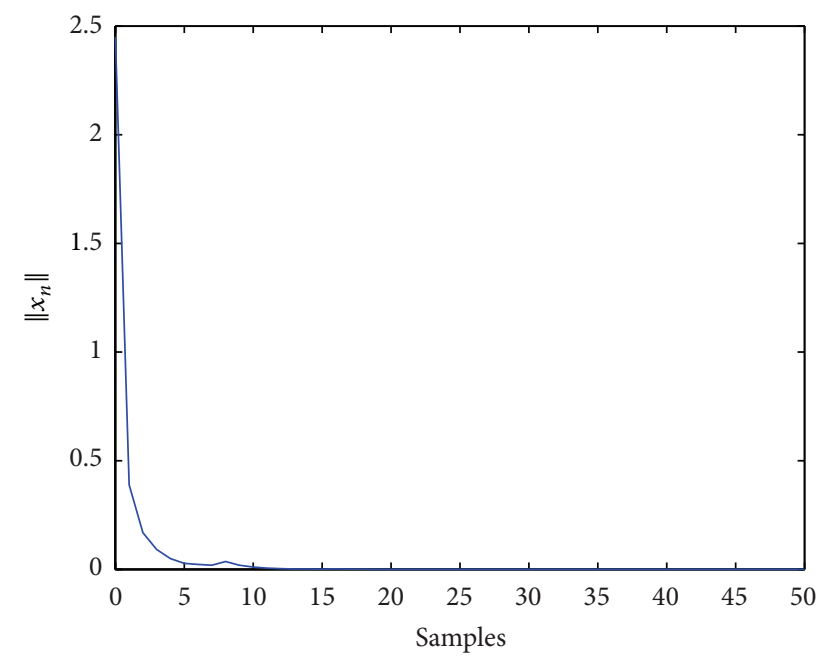

FIGURE 3: Evolution of the norm of the state for the first experiment.
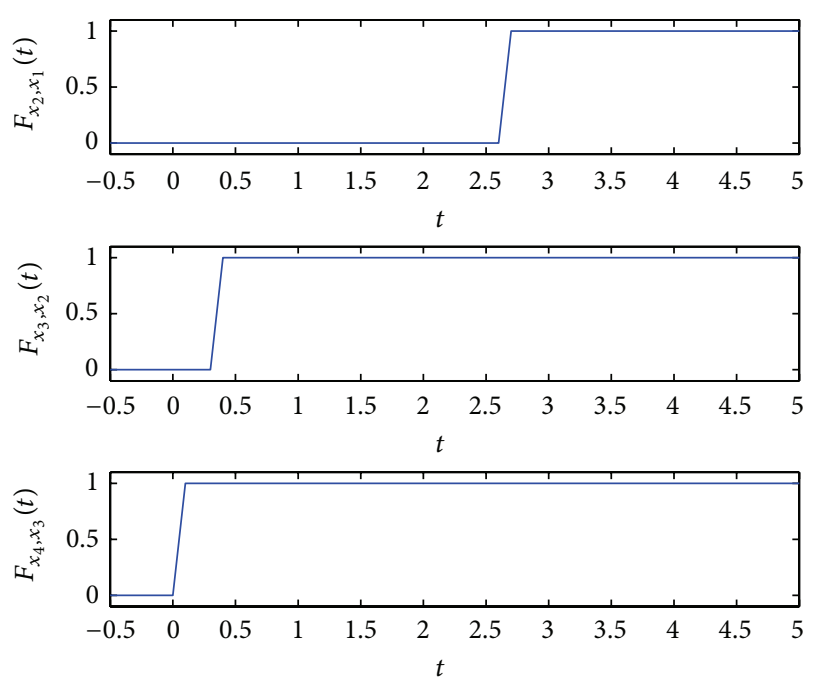

FIgURE 4: Evolution of $F_{x_{n+1}, x_{n}}(t)$ as $n$ increases for the first experiment.

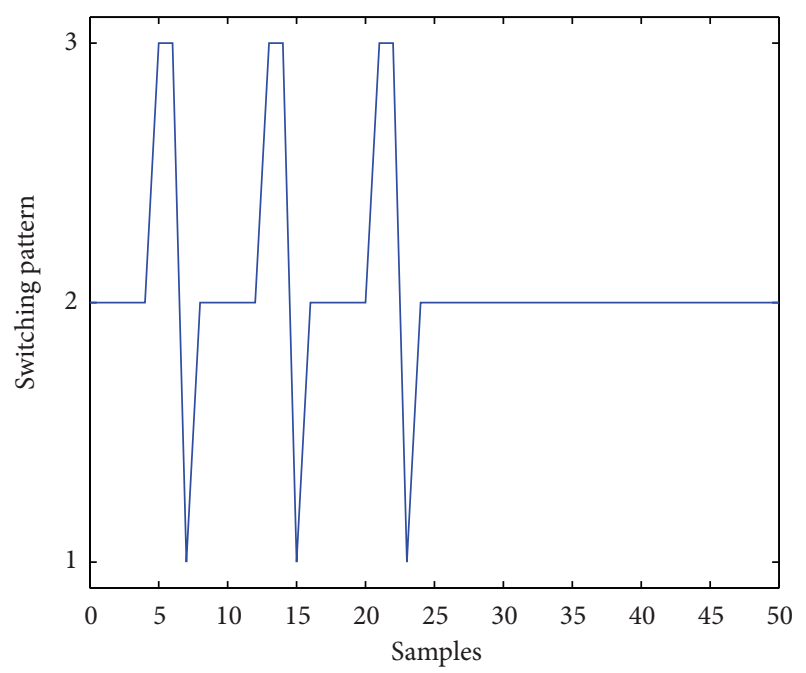

FIGURE 5: Switching map for the second experiment.

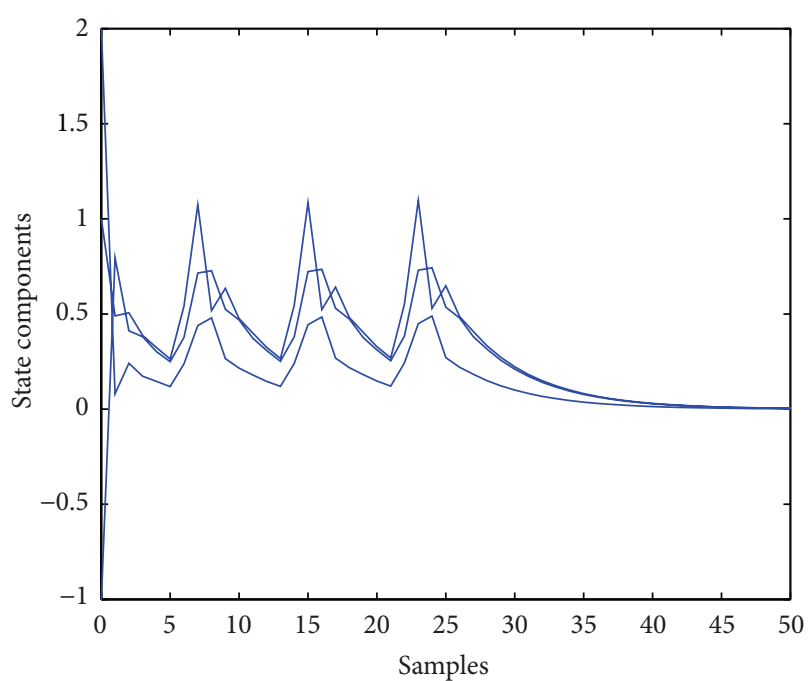

FIGURE 6: Evolution of the state variables in the second experiment.

$\varepsilon=0.2$ and set the perturbation matrices in such way that $\left\|F_{i}\right\|_{2}=\sqrt{\lambda_{\text {max }}\left(F^{T} F\right)} \leq \varepsilon$ for $i=1,2,3$. Thus, let them be

$$
\begin{aligned}
& F_{1}=\left(\begin{array}{ccc}
0.01 & 0.02 & -0.01 \\
0.02 & -0.08 & -0.01 \\
-0.01 & 0.18 & -0.01
\end{array}\right), \\
& F_{2}=\left(\begin{array}{ccc}
-0.01 & 0.18 & -0.01 \\
0.01 & 0.01 & 0.02 \\
0.02 & -0.08 & -0.01
\end{array}\right), \\
& F_{3}=\left(\begin{array}{ccc}
0.02 & -0.08 & -0.01 \\
-0.01 & 0.18 & -0.01 \\
0.01 & 0.02 & -0.01
\end{array}\right) .
\end{aligned}
$$




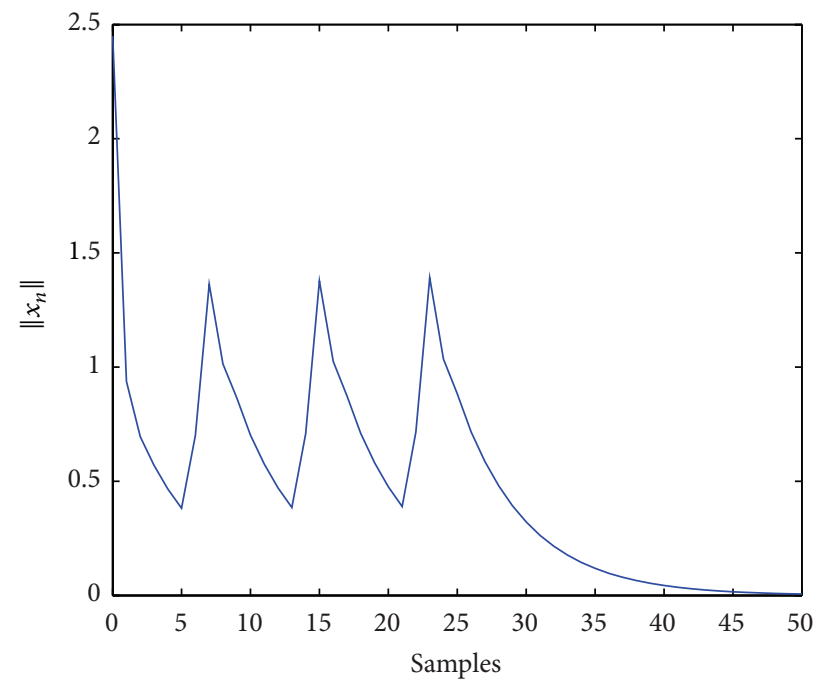

Figure 7: Evolution of the norm of the state in the second experiment.
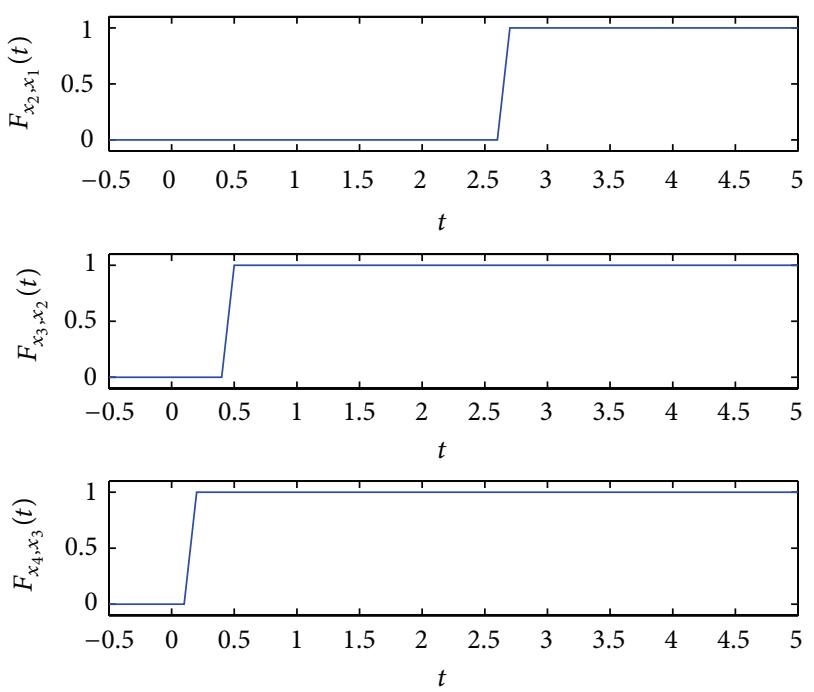

Figure 8: Evolution of $F_{x_{n+1}, x_{n}}(t)$ as $n$ increases for the second experiment.

The perturbation matrices are generated by swapping a file for each subsystem. Therefore, $\left\|F_{1}\right\|_{2}=\left\|F_{2}\right\|_{2}=\left\|F_{3}\right\|_{2}=0.1987$ near the upper bounding value of $\varepsilon=0.2$. Under these circumstances, the worst-case operator $A_{1}+\varepsilon I$ is still contractive, $A_{2}+\varepsilon I$ is nearly nonexpansive and noncontractive, and $A_{3}-\varepsilon I$ is always expansive providing different dynamics to each of the subsystems. With this setup, we will perform three simulation experiments to illustrate the diversity of dynamical behaviours that can be achieved by modifying the switching law. The initial condition in all experiments is $x_{0}=$ $\left[\begin{array}{lll}1 & -1 & 2\end{array}\right]^{T}$.

Experiment 1. The switching law never stops and the first subsystem prevails over the other two. The switching law is selected to be periodic, with a period of 8 samples according to the pattern displayed in Figure 1. This means that in one

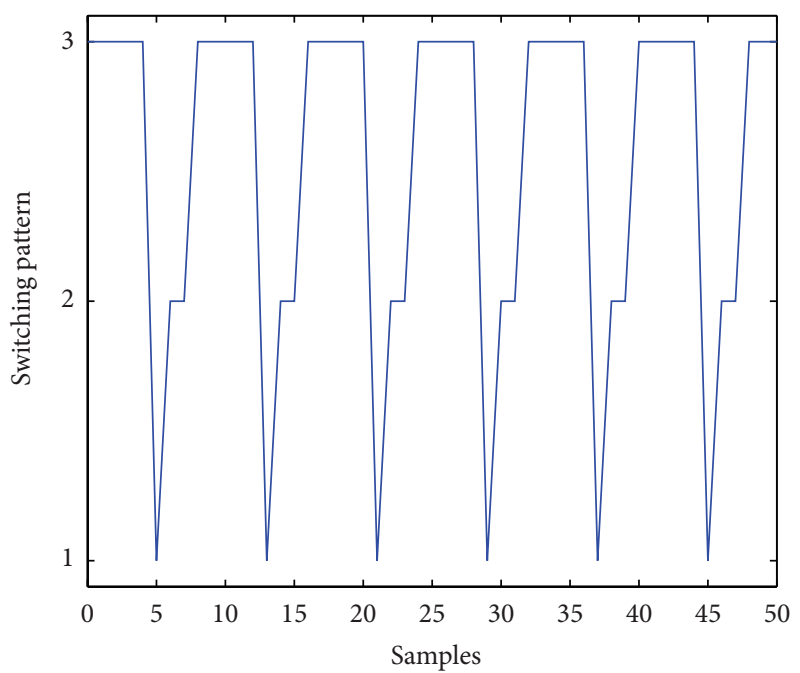

FIGURE 9: Switching patterns for the third experiment.

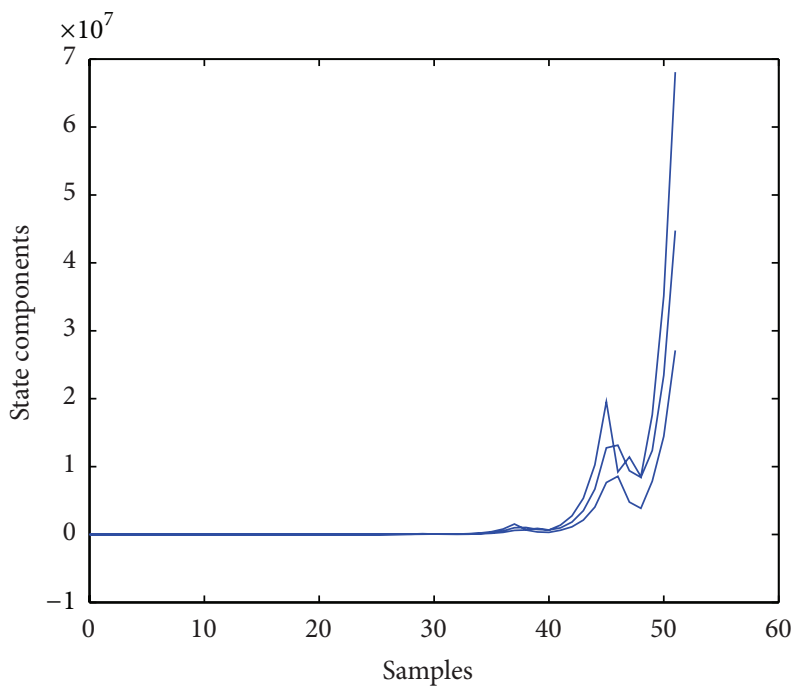

FIGURE 10: Evolution of the state variables for the third experiment.

period we have $5=\gamma_{1}>\gamma_{2}+\gamma_{3}=2+1$; that is, the time interval within which the first subsystem is active is larger than the sum of the intervals corresponding to the other subsystems. Thus, Theorem 17 ensures that the sequence of iterates is probabilistically bounded and converges to the fixed point $x=0$ as Figure 2 displays for the state components and Figure 3 for the norm of the state. Moreover, Figure 4 shows how $F_{x_{n+1}, x_{n}(t)}$ converges to the Heaviside function $H(t)$ as the iteration variable $n$ increases.

Experiment 2. The switching map converges in finite time to the second subsystem, which is nearly to be nonexpansive and noncontractive. Thus, the switching map is depicted in Figure 5. Figures 6 and 7 show, respectively, the evolution of the state variables and their norm as $n$ increases. As Theorem 17 states, the sequence of iterates is bounded and converges to the fixed point because, due to numerical roundoff errors, the operator $T_{2}$ is slightly contractive. However, 


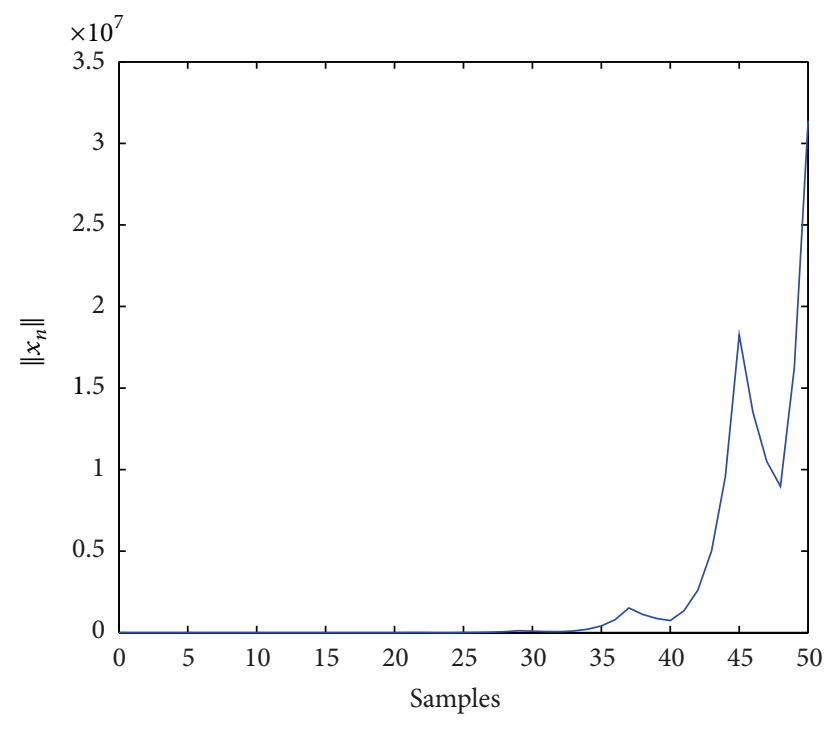

FIGURE 11: Norm of the state for the third experiment.
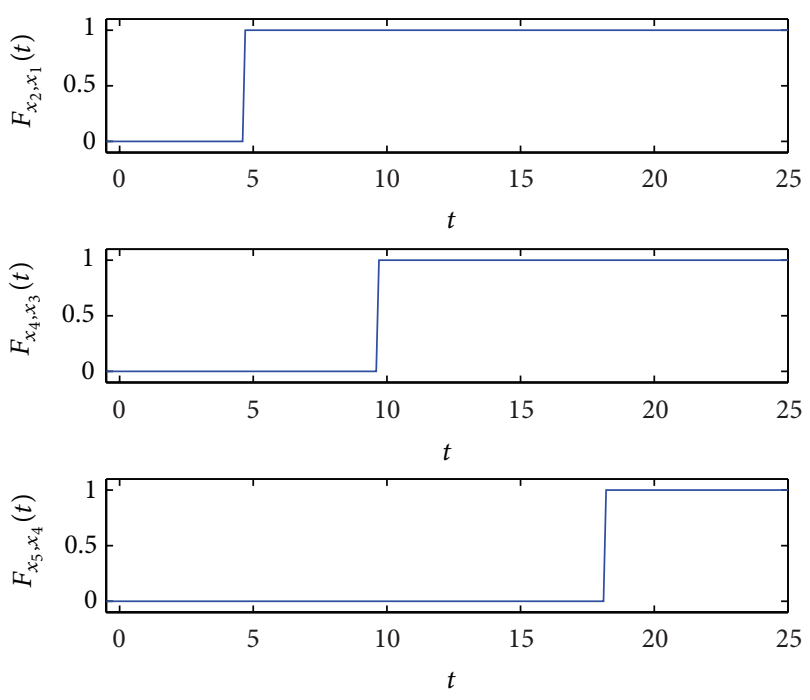

FIGURE 12: Evolution of $F_{x_{n+1}, x_{n}}(t)$ as $n$ increases for the third experiment.

it converges at a lower rate than in Experiment 1 since the operator $T_{2}$ is very nearly to be noncontractive and nonexpansive. In this case, $F_{x_{n+1}, x_{n}}(t)$ also converges to the Heaviside function $H(t)$ as the iteration variable $n$ increases, as it can be noticed in Figure 8 .

Experiment 3. The switching law never stops and the third subsystem prevails over the other two. A periodic switching signal is considered again with $\gamma_{3}>\gamma_{1}+\gamma_{2}$ in a period. Figure 9 gives the switching law. In addition, Figures 10 and 11 , respectively, display the evolution of the state variables and their norm as $n$ increases. As we could have expected from Theorem 17, they are not bounded and diverge asymptotically since the expansive operator dominates the period of the switching pattern. Contrarily to the previous experiments, $F_{x_{n+1}, x_{n}}(t)$ converges now to the identically null function, since, as $n$ increases, the Heaviside function $H(t)$ is displaced to the right, as it is represented in Figure 12.

\section{Conflict of Interests}

The authors declare that they do not have competing interests.

\section{Authors' Contribution}

The authors declare that all the authors contributed equally to all of the parts of this paper.

\section{Acknowledgments}

The authors are very grateful to the Spanish Government for Grants DPI2012-30651 and DPI2013-47825-C3-1-R and also to the Basque Government and UPV/EHU for Grants IT37810, SAIOTEK SPE13UN039, and UFI 2011/07.

\section{References}

[1] S. Kumar and S. Chauhan, "Fixed points of expansion mappings in Menger spaces using weak compatibility," The Mathematics Student, vol. 82, no. 2-4, pp. 157-164, 2013.

[2] R. J. Egbert, "Products and quotients of probabilistic metric spaces," Pacific Journal of Mathematics, vol. 24, pp. 437-455, 1968.

[3] V. M. Sehgal and A. T. Bharucha-Reid, "Fixed points of contraction mappings on probabilistic metric spaces," Mathematical Systems Theory, vol. 6, pp. 97-102, 1972.

[4] D. Miheț, "Altering distances in probabilistic Menger spaces," Nonlinear Analysis: Theory, Methods \& Applications, vol. 71, no. 7-8, pp. 2734-2738, 2009.

[5] C.-M. Chen, "Fixed point theorems of generalized cyclic orbital Meir-Keeler contractions," Fixed Point Theory and Applications, vol. 2013, article 91, 2013.

[6] M. A. Al-Thagafi and N. Shahzad, "Convergence and existence results for best proximity points," Nonlinear Analysis, Theory, Methods \& Applications, vol. 70, no. 10, pp. 3665-3671, 2009.

[7] S. Rezapour, M. Derafshpour, and N. Shahzad, "Best proximity points of cyclic $\varphi$-contractions on reflexive Banach spaces," Fixed Point Theory and Applications, vol. 2010, Article ID 946178, 7 pages, 2010.

[8] C. Di Bari, T. Suzuki, and C. Vetro, "Best proximity points for cyclic Meir-Keeler contractions," Nonlinear Analysis: Theory, Methods \& Applications, vol. 69, no. 11, pp. 3790-3794, 2008.

[9] S. Rezapour, M. Derafshpour, and N. Shahzad, "On the existence of best proximity points of cyclic contractions," Advances in Dynamical Systems and Applications, vol. 6, no. 1, pp. 33-40, 2011.

[10] N. Hussain, M. A. Kutbi, and P. Salimi, "Best proximity point results for modified $\alpha-\psi$ proximal rational contractions," Abstract and Applied Analysis, vol. 2013, Article ID 927457, 14 pages, 2013.

[11] M. Gabeleh, "Best proximity point theorems via proximal nonself mappings," Journal of Optimization Theory and Applications, vol. 164, no. 2, pp. 565-576, 2015.

[12] M. De la Sen, R. P. Agarwal, and A. Ibeas, "Results on proximal and generalized weak proximal contractions including the case 
of iteration-dependent range sets," Fixed Point Theory and Applications, vol. 2014, article 169, 2014.

[13] S. Manro and Sumitra, "Common new fixed point theorem in modified intuitionistic fuzzy metric spaces using implicit relation," Applied Mathematics, vol. 4, no. 9, pp. 27-31, 2013.

[14] A. F. Roldán-López-de-Hierro, M. de la Sen, J. MartínezMoreno, and C. Roldán-López-de-Hierro, "An approach version of fuzzy metric spaces including an ad hoc fixed point theorem," Fixed Point Theory and Applications, vol. 2015, article 33, 2015.

[15] M. De la Sen and E. Karapınar, "Some results on best proximity points of cyclic contractions in probabilistic metric spaces," Journal of Function Spaces, vol. 2015, Article ID 470574, 11 pages, 2015.

[16] M. De la Sen, "On fixed and best proximity points of cyclic C-contractions in probabilistic complete metric and Banach spaces," Bulletin of the Malaysian Mathematical Sciences Society, 2015.

[17] S. A. Sahab, M. S. Khan, and S. Sessa, "A result in best approximation theory," Journal of Approximation Theory, vol. 55, no. 3, pp. 349-351, 1988.

[18] M. De la Sen and E. Karapinar, "On a cyclic Jungck modified TS-iterative procedure with application examples," Applied Mathematics and Computation, vol. 233, pp. 383-397, 2014.

[19] A. R. Khan, V. Kumar, and N. Hussain, "Analytical and numerical treatment of Jungck-type iterative schemes," Applied Mathematics and Computation, vol. 231, pp. 521-535, 2014.

[20] J.-Z. Xiao, X.-H. Zhu, and X.-Y. Liu, "An alternative characterization of probabilistic Menger spaces with H-type triangular norms," Fuzzy Sets and Systems, vol. 227, pp. 107-114, 2013.

[21] T. Dinevari and M. Frigon, "Fixed point results for multivalued contractions on a metric space with a graph," Journal of Mathematical Analysis and Applications, vol. 405, no. 2, pp. 507517, 2013.

[22] M. de la Sen and A. Ibeas, "On the stability properties of linear dynamic time-varying unforced systems involving switches between parameterizations from topologic considerations via graph theory," Discrete Applied Mathematics, vol. 155, no. 1, pp. 7-25, 2007.

[23] M. D. Sen, S. Alonso-Quesada, and A. Ibeas, "On the asymptotic hyperstability of switched systems under integral-type feedback regulation Popovian constraints," IMA Journal of Mathematical Control and Information, vol. 32, no. 2, pp. 359-386, 2015.

[24] M. De la Sen and A. Ibeas, "Properties of convergence of a class of iterative processes generated by sequences of self-mappings with applications to switched dynamic systems," Journal of Inequalities and Applications, vol. 2014, article 498, 2014.

[25] M. de la Sen and A. Ibeas, "On the global asymptotic stability of switched linear time-varying systems with constant point delays," Discrete Dynamics in Nature and Society, vol. 2008, Article ID 231710, 31 pages, 2008.

[26] C. Duan and F. Wu, "New results on switched linear systems with actuator saturation," International Journal of Systems Science, 2014.

[27] M. Rajchakit and G. Rajchakit, "Exponential stability of stochastic hybrid systems with nondifferentiable and interval time-varying delay," International Journal of Pure and Applied Mathematics, vol. 95, no. 1, pp. 79-88, 2014.

[28] M. De la Sen, "Linking contractive self-mappings and cyclic Meir-Keeler contractions with Kannan self-mappings," Fixed Point Theory and Applications, vol. 2010, Article ID 572057, 2010. 


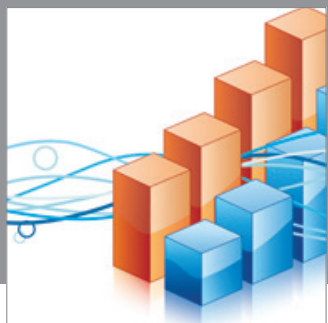

Advances in

Operations Research

mansans

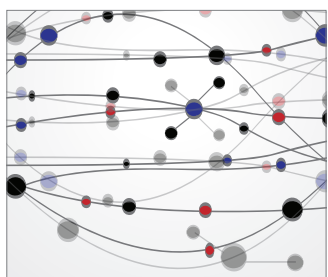

The Scientific World Journal
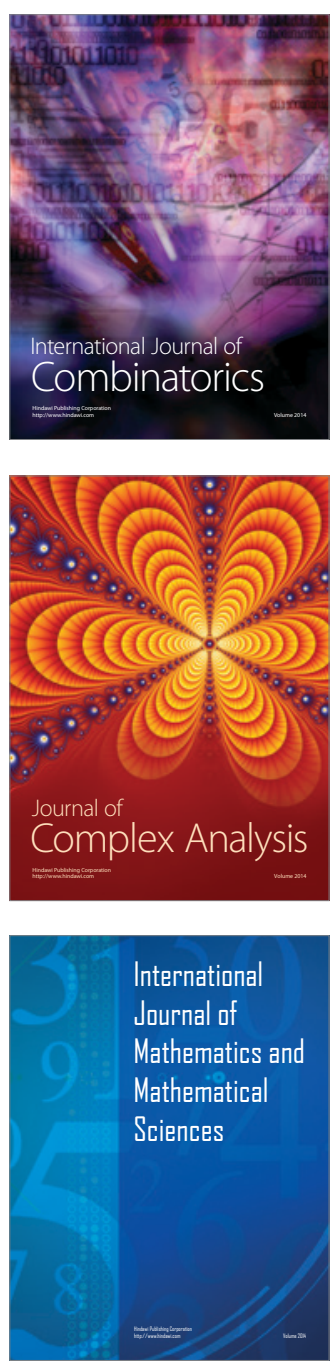
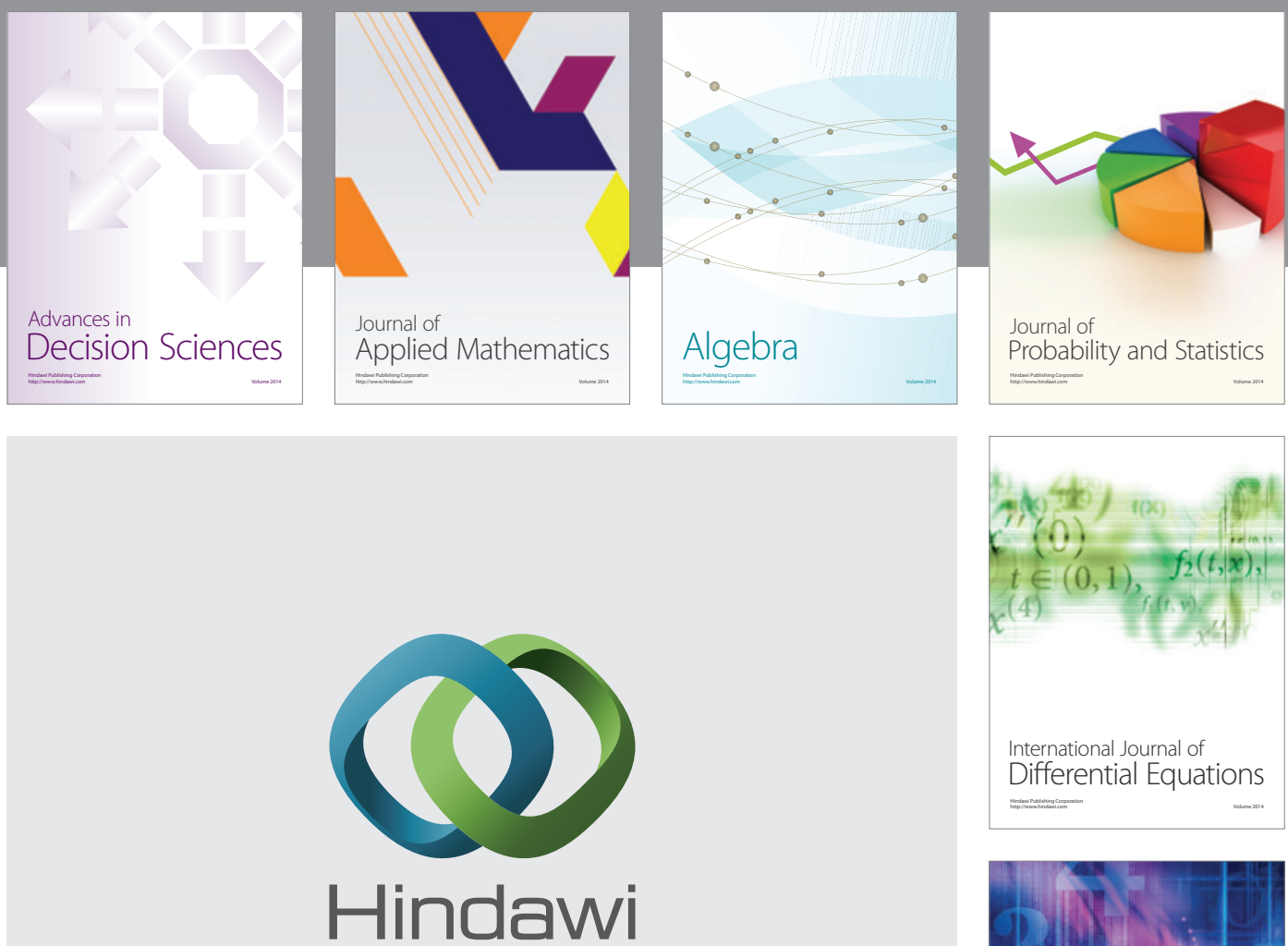

Submit your manuscripts at http://www.hindawi.com
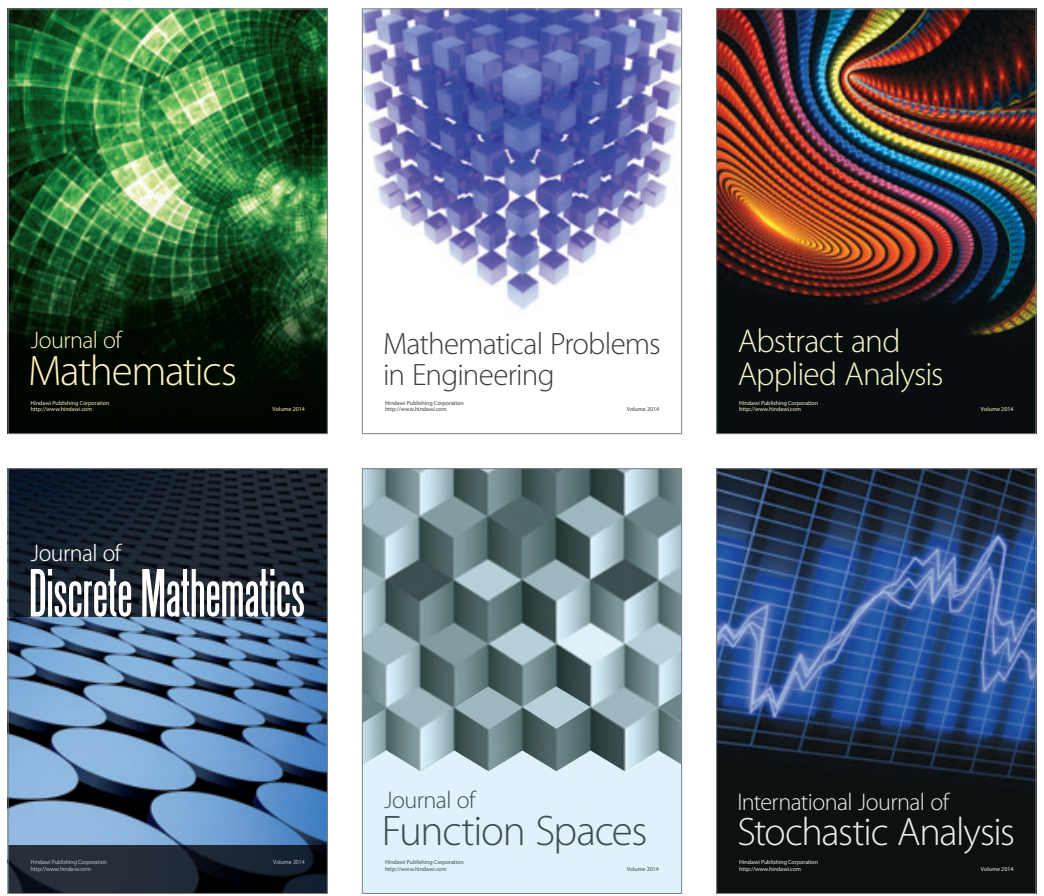

Journal of

Function Spaces

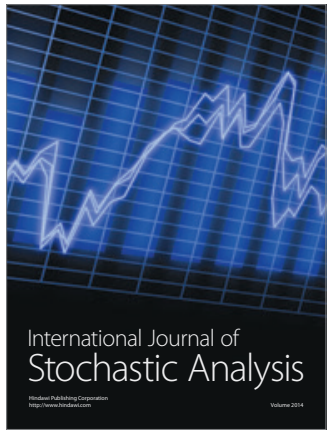

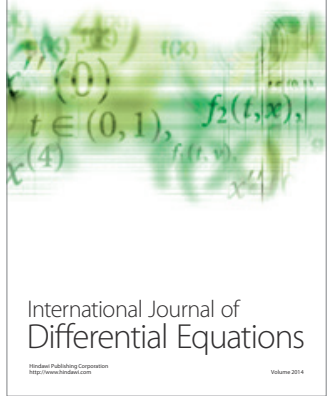
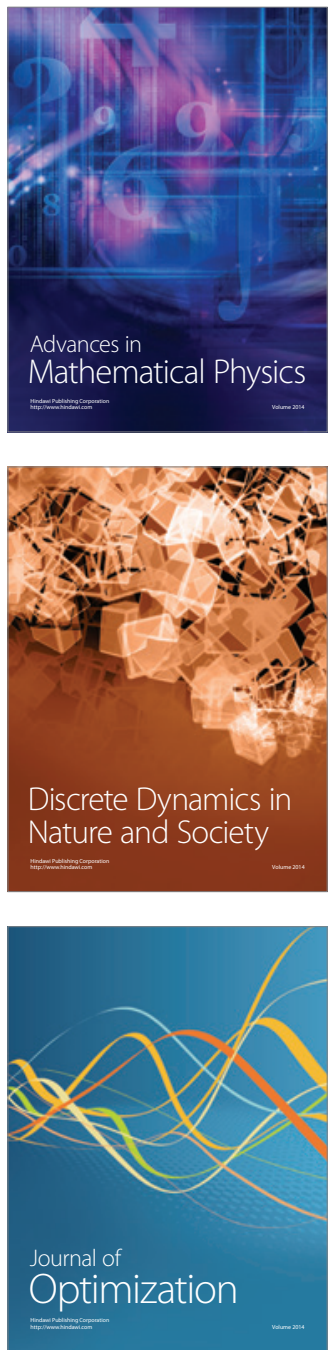